\title{
PORTFOLIO OPTIMIZATION
}

\author{
BY \\ RENÉ SCHNIEPER \\ Zurich Insurance Company, Reinsurance
}

KEYWORDS

Reinsurance, retentions, non linear optimization, insurance risk, financial risk, Markowitz's portfolio selection method, CAPM.

\begin{abstract}
Based on the profit and loss account of an insurance company we derive a probabilistic model for the financial result of the company, thereby both assets and liabilities are marked to market. We thus focus on the economic value of the company.

We first analyse the underwriting risk of the company. The maximization of the risk return ratio of the company is derived as optimality criterion. It is shown how the risk return ratio of heterogeneous portfolios or of catastrophe exposed portfolios can be dramatically improved through reinsurance. The improvement of the risk return ratio through portfolio diversification is also analysed.

In section 3 of the paper we analyse the loss reserve risk of the company. It is shown that this risk consists of a loss reserve development risk and of a yield curve risk which stems from the discounting of the loss reserves. This latter risk can be fully hedged through asset liability matching.

In section 4 we derive our general model. The portfolio of the company consists of a portfolio of insurance risks and of a portfolio of financial risks. Our model allows for a simultaneous optimization of both portfolios of risks. A theorem is derived which gives the optimal retention policy of the company together with its optimal asset allocation.

Some of the material presented in this paper is taken from Schnieper, 1997. It has been repeated here in order to make this article self contained.
\end{abstract}




\section{TABLE OF CONTENTS}

$\begin{array}{ll}\text { 1. Introduction } & 197\end{array}$

2. Underwriting Risk 198

$\begin{array}{lr}\text { 2.1. Simplified Model } & 198\end{array}$

2.2. Optimality Criterion 199

2.3. Portfolio Heterogeneity 203

2.4. Catastrophe Exposure 206

$\begin{array}{ll}\text { 2.5. Portfolio Diversification } & 208\end{array}$

3. Loss Reserve Risk 213

3.1. Individual Accident Year 213

3.2. Different Accident Years 216

4. General Model Including Asset Risk 219

$\begin{array}{ll}\text { 4.1. Optimality Criterion } & 219\end{array}$

4.2. Portfolio Optimization 222

4.3. Insurance Risk and Financial Risk 235

$\begin{array}{ll}\text { 4.4. Realistic Example } & 237\end{array}$

5. Comparison with Other Results in Finance Theory 241

5,1. Markowitz's Portfolio Selection Method 241

5.2. CAPM 242 


\section{INTRODUCTION}

The profit and loss account of an insurance company typically details the following income items:

-- earned premiums (net of premiums for outwards reinsurance),

- investment income,

- realized capital gains,

and the following expenditure positions:

- incurred claims (net of reinsurance recoveries),

- expenses,

- dividends to policyholders,

- dividends to shareholders.

We assume that the accounts of the company are on an accident year basis. Any other commonly used basis (e.g. underwriting year) can be dealt with after some minimal changes. We shall some times refer to the financial year which is the period covered by the company's accounts.

We split the premium into its different components;

- pure risk premium,

- loading for expenses,

- loading for profit.

We split incurred claims into the following two components:

- incurred claims pertaining to the current accident year

- changes in claim amounts in respect of claims pertaining to previous accident years.

We also take unrealized capital gains into account as an income item.

We make the following simplifying assumptions:

-- expenses and loading for expenses are identical and therefore cancel out;

- dividends to policyholders are accounted for as claims,

- we are interested in the change in value of the surplus of the company before dividend to shareholders. We therefore ignore this item,

- the period under consideration is the financial year of the company. This is an arbitrary assumption. We could take any other period e.g. a quarter or a multi year period corresponding to the planing horizon of the company,

- payments pertaining to a given period are made at the end of the period,

- the premium written in a given period is earned in that period, i.e. the company has no unearned premium reserves. (This assumption can be dropped at the cost of a slight increase in the model complexity. The interest rate risk pertaining to the unearned premium reserves would be treated in a similar way as the interest rate risk pertaining to the loss reserves. Since the former is much less material than the latter, we have chosen to ignore it.) 
We make the following model assumptions:

1. All random variables appearing in the model have finite second order moments.

2. The pure risk premium is the present value of the expected loss payments.

3 . The loss reserves are equal to the present values of expected future loss payments.

4. The discount factors used to assess the pure risk premium and the loss reserves are based on the yield curve as defined by the bond market.

5. The assets of the company are valued at market value.

We introduce the following notation, where random variables are denoted by a tilde:

$\tilde{S} \quad$ total claims amount pertaining to the current accident year

$E(\tilde{S}) \quad$ the mathematical expectation of the above random variable; this is the pure risk premium

$\ell \quad$ the profit loading for assuming the underwriting risk $\tilde{S}$

$\tilde{\Delta} L \quad$ increase in claim amounts in respect of claims pertaining to previous accident years

$\tilde{\Delta} A \quad$ investment income plus realized capital gains plus unrealized capital gains

$u \quad$ capital (economic value) of the company at the beginning of the financial year

$\tilde{\Delta} u \quad$ increase in capital (in economic value) during the financial year, return of the company during the financial year.

The following relation holds true

$$
\tilde{\Delta} u=E(\tilde{S})+\ell-\tilde{S}-\tilde{\Delta} L+\tilde{\Delta} A
$$

$\tilde{S}-E(\tilde{S})$ is referred to as the underwriting risk, $\tilde{\Delta} L-E(\tilde{\Delta} L)$ as the loss reserve risk, $\tilde{\Delta} A-E(\tilde{\Delta} A)$ as the asset risk and $\tilde{\Delta} u-E(\tilde{\Delta} u)$ as to the total risk of the company.

\section{UNDERWRITING RISK}

\subsection{Simplified Model}

We split the assets of the company between a liability fund and a capital fund $A=A_{L}+A_{U}$. This means that some of the assets $\left(A_{L}\right)$ are earmarked to cover the liabilities of the company and the rest of the assets $\left(A_{U}\right)$ match the equity of the company. Since in this section we focus on the underwriting risk, we assume that there is no loss reserve risk and no asset risk. To be more specific, we make the following 


\section{Assumptions}

- There is no loss reserve risk, i.e. amount and time of payment in respect of outstanding losses are perfectly known to the company.

- The liability fund, i.e. those assets which cover the liabilities, perfectly match the amounts and maturities of the liabilities. The liabilities are discounted with the discount factors corresponding to the liability fund. As a consequence any change in the yield curve will have a perfectly offsetting effect on $\tilde{\Delta} L$ and $-\Delta \tilde{A}_{L}$.

- The capital fund is invested in the risk free rate of return: $\tilde{\Delta} A_{U}=\rho_{0} u$.

The total return of the company now is

$$
\tilde{\Delta} u=E(\tilde{S})+\ell-\tilde{S}-\tilde{\Delta} L+\tilde{\Delta} A_{L}+\tilde{\Delta} A_{U}=E(\tilde{S})+\ell-\tilde{S}+\rho_{0} u
$$

\subsection{Optimality Criterion}

The objective of the present article is to provide a method to optimize the portfolio of the company. We first define and discuss the optimality criterion. The owners of the company are interested in the excess return on equity provided by the insurance portfolio

$$
\tilde{\delta}(u)=\frac{\tilde{\Delta} u-\rho_{0} u}{u}
$$

Let

$$
E(\tilde{S})-\ell-\tilde{S}=\sum_{i=1}^{m} E\left(\tilde{X}_{i}\right)+\ell_{i}-\tilde{X}_{i}
$$

be a breakdown of the portfolio into $m$ individual risks (policies, lines of business, customer segments, etc.). The company manages its portfolio by defining for each risk $\tilde{X}_{i}-E\left(\tilde{X}_{i}\right)$ the share $\alpha_{i} \in[0,1]$ it wants to retain and by ceding $\left(1-\alpha_{i}\right)\left(\tilde{X}_{i}-E\left(\tilde{X}_{i}\right)\right)$ to its reinsurers. It is assumed that the company also cedes a proportional share of the corresponding profit $\left(1-\alpha_{i}\right) \ell_{i}$ to its reinsurers. The return of the net retained portfolio is thus

$$
\tilde{\Delta} u_{n e t}=\sum_{i=1}^{m} \alpha_{i}\left(E\left(\tilde{X}_{i}\right)+\ell_{i}-\tilde{X}_{i}\right)+\rho_{0} u
$$

and the corresponding excess return on equity is

$$
\tilde{\delta}_{\alpha}(u)=\frac{\tilde{\Delta} u_{n e t}-\rho_{0} u}{u}=\sum_{i=1}^{m} \alpha_{i} \frac{E\left(\tilde{X}_{i}\right)+\ell_{i}-\tilde{X}_{i}}{u}
$$


We introduce the following notation

$$
\mu_{\alpha}(u)=E\left(\tilde{\delta}_{\alpha}(u)\right) \quad \sigma_{\alpha}^{2}(u)=\operatorname{Var}\left(\tilde{\delta}_{\alpha}(u)\right) .
$$

We have now to define the criterion according to which the company optimizes its portfolio. The approach is the same as Markowitz's mean variance method. (See H. Panjer et al., 1998.) It is assumed that the owners of the company have two objectives:

- maximization of the expected value $\mu_{\alpha}(u)$ of the company return on equity

- minimization of the risk as measured by $\sigma_{\alpha}^{2}(u)$.

According to their preferences, the owners put weights on these conflicting objectives and maximize

$$
2 \tau \mu_{\alpha}(u)-\sigma_{\alpha}^{2}(u), \quad \text { with } \tau \geq 0 .
$$

The parameter $\tau$ is called the risk tolerance.

Note that the total investment constraint of the Markowitz Model $\left(\sum_{i=1}^{m} \alpha_{i}=1\right)$ is meaningless in the present framework and has been dropped.

We first assume that the amount of equity of the company, $u$ is given. The set of all points in the $(\mu, \sigma)$ diagram, which correspond to efficient portfolios is called the efficient frontier. The efficient frontier is convex, and piecewise hyperbolic. Because there exists a riskless investment, the first piece of the efficient frontier is linear. (See H. Panjer et al., 1998.)

\section{Example}

We assume that there are two uncorrelated risks with expected profit $\ell_{1}$ and $\ell_{2}$ respectively and standard deviation $\sigma_{1}$ and $\sigma_{2}$ respectively. We introduce the following notation

$$
\lambda_{i}=\frac{\ell_{i}}{\mathfrak{u}} \quad \text { and } \quad \tau_{i}=\frac{\sigma_{i}}{\mathfrak{u}} \quad i=1,2 .
$$

We have

$$
\begin{aligned}
& \mu_{\alpha}(u)=\alpha_{1} \lambda_{1}+\alpha_{2} \lambda_{2} \\
& \sigma_{\alpha}^{2}(u)=\alpha_{1}^{2} \tau_{1}^{2}+\alpha_{2}^{2} \tau_{2}^{2}
\end{aligned}
$$

The objective is

$$
2 \tau \mu_{\alpha}(u)-\sigma_{\alpha}^{2}(u)=\max _{\underline{\alpha} \in \beta} ! \quad \text { with } \quad \beta=\left\{\underline{\alpha} \mid \alpha_{1}, \alpha_{2} \in[0,1]\right\}
$$

which leads to the following unconstrained optimum

$$
\alpha_{i}=\tau \frac{\lambda_{i}}{\tau_{i}^{2}} \quad i=1,2
$$


Without any loss of generality we assume

$$
\frac{\lambda_{1}}{\tau_{1}^{2}} \geq \frac{\lambda_{2}}{\tau_{2}^{2}}
$$

and we make the following case distinction:

1. $\tau \leq \frac{\tau_{1}^{2}}{\lambda_{1}}$

In that case $\alpha_{1}$ and $\alpha_{2}$ are as above and

$\mu_{\alpha}=\tau\left(\frac{\lambda_{1}}{\tau_{1}^{2}}+\frac{\lambda_{2}}{\tau_{2}^{2}}\right), \sigma_{\alpha}^{2}=\tau^{2}\left(\frac{\lambda_{1}^{2}}{\tau_{1}^{2}}+\frac{\lambda_{2}^{2}}{\tau_{2}^{2}}\right)$.

Hence $\left(\mu_{\alpha}, \sigma_{\alpha}\right)$ describes a straight line as $\tau$ varies.

2. $\tau \in\left[\frac{\tau_{1}^{2}}{\lambda_{1}}, \frac{\tau_{2}^{2}}{\lambda_{2}}\right]$

In that case $\alpha_{1}=1$ and $\alpha_{2}=\tau \frac{\lambda_{2}}{\tau_{2}^{2}}$ and $\mu_{\alpha}=\lambda_{1}+\tau \frac{\lambda_{2}^{2}}{\tau_{2}^{2}}, \sigma_{\alpha}^{2}=\sigma_{1}^{2}+\tau^{2} \frac{\lambda_{2}^{2}}{\tau_{2}^{2}}$ and $\left(\mu_{\alpha}, \sigma_{\alpha}\right)$ describes a hyperbole as $\tau$ varies.

3. $\tau \geq \frac{\tau_{2}^{2}}{\lambda_{1}}$

In that case $\alpha_{1}=\alpha_{2}=1$ and $\mu_{\alpha}=\lambda_{1}+\lambda_{2}, \sigma_{\alpha}^{2}=\sigma_{1}^{2}+\sigma_{2}^{2}$ and this segment of the efficient frontier degenerates to a single point.

We now let the amount of equity of the company, $u$ vary. We have

$$
\begin{aligned}
& \mu_{\alpha}(u)=E\left(\tilde{\delta}_{\alpha}(u)\right)=\frac{\sum_{i=1}^{m} \alpha_{i} \ell_{i}}{u}=\frac{R(\underline{\alpha})}{u} \\
& \sigma_{\alpha}^{2}(u)=\operatorname{Var}\left(\tilde{\delta}_{\alpha}(u)\right)=\frac{\sum_{i, j} \alpha_{i} \alpha_{j} \sigma_{i j}^{2}}{u^{2}}=\frac{V(\underline{\alpha})}{u^{2}}
\end{aligned}
$$

where $\sigma_{i, j}^{2}=\operatorname{Cov}\left(\tilde{X}_{i}, \tilde{X}_{j}\right)$. Hence $\mu_{\alpha}(u)=\frac{R(\underline{\alpha})}{u}, \sigma_{\alpha}(u)=\frac{\dot{V}(\underline{\alpha})^{\frac{1}{2}}}{u}$.

Thus if $P$ is a point on the efficient frontier as defined above - i.e. on the basis of a fixed amount of equity - any point on the straight line $0 P$ can be reached through a proper choice of the amount of equity $u$. It is therefore natural to start the optimization process with the following requirement

1. $\frac{R(\underline{\alpha})}{(V(\underline{\alpha}))^{\frac{1}{2}}}=\max _{\underline{\alpha} \in \beta}$ ! with $\beta=\left\{\underline{\alpha} \mid \alpha_{i} \in[0,1]\right.$ all $\left.i\right\}$

the above requirement amounts to a maximization of the risk return ratio or, in the terminology of financial economics, of Sharpe's ratio. In 
general, the above ratio is maximized for a whole set of admissible values of $\alpha$. Let $\beta_{1}$ denote the set of those values. It is reasonable to make the following additional requirement

$$
\sum_{i=1}^{m} \alpha_{i} \ell_{i}=\max _{\underline{\alpha} \in \beta_{1}} !
$$

This amounts to maximizing the net expected profit.

Let $\underline{\alpha}_{M}$ denote the net retentions for which the above requirement is satisfied. Let

$$
R=R\left(\underline{\alpha}_{M}\right) \text { and } \quad V=V\left(\underline{\alpha}_{M}\right) .
$$

The optimal amount of equity is now defined by the following requirement

2. $2 \tau \frac{R}{u}-\frac{V}{u^{2}}=\max _{u}$ !

which leads to the following optimal amount of equity

$$
u=\tau^{-1} \frac{V}{R}
$$

\section{Remarks}

1. Whilst the present optimization method is based on the same objective function as Markowitz's mean variance method, there are however major differences between the two methods. First, the portfolio to be optimized consists of a set of insurance risks rather than financial assets. (Later we shall optimize a combined portfolio of insurance risks and financial assets.) This leads to a different set of constraints. In particular the total investment constraint $\left(\sum \alpha_{i}=1\right)$ is meaningless and has been dropped. Second, in addition to optimizing the composition of the portfolio, the company can also decide on the amount of equity it needs to support the business. This additional degree of freedom leads to a different efficient frontier than in the Markowitz framework.

2. One of the drawbacks of the above method is that it only takes into account the first two moments of the distribution of the risks in the portfolio. In the case of insurance risks which are typically skewed and leptocurtic, this is a serious limitation. In the remainder of this section we shall nevertheless analyze a few insurance optimization problems with the help of the above method. It is felt that this parallel between insurance and finance is of interest in spite of the above mentioned limitations. Within the framework of our general model (introduced in section 4) we optimize a combined portfolio of insurance risks and of risky financial assets. Since the insurance risks entering into the portfolio are net of reinsurance, it is not unreasonable to assume that the distribution of returns is close to multivariate normal. 
We now turn to the problem of allocating capital to individual risks. Let $\tilde{\Delta} u=\sum_{i=1}^{n} \tilde{X}_{i}$ be any split of the total risk of the company into individual risks. The capital is proportional to

$$
\operatorname{Var}(\tilde{\Delta} u)=\sum_{i=1}^{n} \operatorname{Cov}\left(\tilde{X}_{i}, \tilde{\Delta} u\right)
$$

It is thus fair to allocate to each risk $\tilde{X}_{i}$ an amount of capital $u_{i}$, which is proportional to the contribution of that risk to the overall volatility of the result of the company: $u_{i}=k \cdot \operatorname{Cov}\left(\tilde{X}_{i}, \tilde{\Delta} u\right)$. Since $u=\sum_{i=1}^{n} u_{i}$ we obtain

$$
u_{i}=u \cdot \frac{\operatorname{Cov}\left(\tilde{X}_{i}, \tilde{\Delta} u\right)}{\operatorname{Var}(\tilde{\Delta} u)}
$$

The excess return which the company expects to achieve for assuming the risk $\sigma(\Delta u)$ is equal to $\left(\rho-\rho_{0}\right) u$, where $\rho_{0}$ denotes the risk free rate of return. It is fair to split the excess return proportionally to the capital.

\section{Definition}

The fair loading of risk $\tilde{X}_{i}$ is

$$
\left(\rho-\rho_{0}\right) u_{i}=\left(\rho-\rho_{0}\right) \cdot u \cdot \frac{\operatorname{Cov}\left(\tilde{X}_{i}, \tilde{\Delta} u\right)}{\operatorname{Var}(\tilde{\Delta} u)}
$$

It is equal to the cost of the capital needed for assuming risks $\tilde{X}_{i}$.

We assume that the company is a price taker, the fair loading is thus not a way to compute prices but a way to define benchmarks. In general there will be cross-subsidies. Certain risks well have a higher expected profit than the fair loading, others will have a lower expected profit. Later we show that if the portfolio of risks is optimized in an unconstrained way, the actual loading of each risk is equal to the fair loading. This is a further justification for our way of allocating capital to individual risks.

We now turn to the problem of maximizing the underwriting risk return ratio. Assuming that the loadings of individual risks are given there are two main possibilities to increase the above ratio: combining risks in a portfolio and buying reinsurance. We illustrate the impact of reinsurance and the portfolio effect on the risk return ratio.

\subsection{Portfolio Heterogeneity}

Let $\tilde{X}_{1}, \tilde{X}_{2}, \ldots, \tilde{X}_{n}$ be the uncorrelated risks of a portfolio $\tilde{S}_{=} \sum_{i=1}^{n} \tilde{X}_{i}$. Let $\ell_{i}$ denote the loading of risk $i$ and $\sigma_{i}^{2}$ its variance. We have thus

$$
\ell=\sum \ell_{i} \text { and } \sigma(\tilde{S})=\left(\sum \sigma_{i}^{2}\right)^{\frac{1}{2}}
$$


Let us assume that for each individual risk $i$ the company keeps a share $\alpha_{i}$ for its own account and cedes a share $\left(1-\alpha_{i}\right)$ to its reinsurers.

\section{Theorem}

Under the above assumptions, the choice of $\alpha_{1}, \ldots, \alpha_{n}$ which maximizes the net underwriting risk return ratio

$$
r_{n e t}=\frac{\sum \alpha_{i} \ell_{i}}{\left(\sum \alpha_{i}^{2} \sigma_{i}^{2}\right)^{\frac{1}{2}}}
$$

is

$$
\alpha_{i}=c \frac{\ell_{i}}{\sigma_{i}^{2}}
$$

where $c$ is some norming constant which must be chosen in such a way that

$$
0 \leq \alpha_{i} \leq 1
$$

for all $i$. With the so defined set of retentions we have

$$
r_{n e t}=\left(\sum_{i} \frac{\ell_{i}^{2}}{\sigma_{i}^{2}}\right)^{\frac{1}{2}}
$$

\section{Proof}

Deriving $r_{\text {net }}$ with respect to $\alpha_{j}$ and setting the derivative equal to 0 we obtain

$$
\begin{gathered}
\frac{\ell_{j}\left(\sum \alpha_{i}^{2} \sigma_{i}^{2}\right)^{\frac{1}{2}}-\left(\sum \alpha_{i} \ell_{i}\right)\left(\sum \alpha_{i}^{2} \sigma_{i}^{2}\right)^{-\frac{1}{2}} \alpha_{j} \sigma_{j}^{2}}{\sum \alpha_{i}^{2} \sigma_{i}^{2}}=0 \\
\ell_{j}\left(\sum \alpha_{i}^{2} \sigma_{i}^{2}\right)=\left(\sum \alpha_{i} \ell_{i}\right) \alpha_{j} \sigma_{j}^{2} \\
\alpha_{j}=\frac{\ell_{j}}{\sigma_{j}^{2}} \frac{\sum \alpha_{i}^{2} \sigma_{i}^{2}}{\sum \alpha_{i} \ell_{i}}=c \frac{\ell_{j}}{\sigma_{j}^{2}}
\end{gathered}
$$

and the value of the optimal $r_{n e t}$ is obtained by plugging the above vaiue of $\alpha_{j}$ into the expression defining $r_{\text {net }}$.

\section{Special case}

Let

$$
\tilde{X}_{i}=\left\{\begin{array}{cll}
L_{i} & \text { with probability } & p \\
0 & \text { with probability } & 1-p
\end{array}\right.
$$

and

$$
\ell_{i}=E\left(\tilde{X}_{i}\right) \lambda=p L_{i} \lambda
$$


we now have

$$
\operatorname{Var}\left(\tilde{X}_{i}\right)=p(1-p) L_{i}^{2} \simeq p L_{i}^{2} \quad \text { for } \quad p<<1
$$

and the optimal retention becomes

$$
\begin{gathered}
\alpha_{i}=c \frac{\ell_{i}}{\sigma_{i}^{2}} \simeq c \frac{p L_{i} \lambda}{p L_{i}^{2}}=\frac{1}{L_{i}} c \lambda \\
\Rightarrow \alpha_{i} L_{i}=c \lambda
\end{gathered}
$$

and the retention of each risk is such that the net monetary amount retained is the same for all risks i.e. the reinsurance arrangement which maximizes the underwriting risk return ratio is a surplus treaty, where the retention is equal to the smallest sum insured.

On a gross basis the risk return ratio is

$$
r=\frac{\sum_{i=1}^{n} L_{i} p \lambda}{\left(\sum_{i=1}^{n} L_{i}^{2} p\right)^{\frac{1}{2}}}=\lambda \sqrt{p} \frac{\sum_{i=1}^{n} L_{i}}{\left(\sum_{i=1}^{n} L_{i}^{2}\right)^{\frac{1}{2}}}
$$

and on a net basis

$$
r_{n e t}=\left(\sum_{i} \frac{\ell_{i}^{2}}{\sigma_{i}^{2}}\right)^{\frac{1}{2}}=\lambda \sqrt{p n}
$$

It is seen that $r_{n e t} \geq r$. The inequality is strict unless all $L_{i}$ 's are equal.

\section{Numerical Example}

Let us assume that there are two types of risks

$$
\tilde{X}_{1}=\left\{\begin{array}{llc}
1 & \text { with probability } & 10^{-3} \\
0 & \text { with probability } & 0.999
\end{array}\right.
$$

and

$$
\tilde{X}_{2}=\left\{\begin{array}{rlc}
100 & \text { with probability } & 10^{-3} \\
0 & \text { with probability } & 0.999
\end{array}\right.
$$

There are $n=10^{5}$ risks of the first type, and $n=10^{3}$ risks of the second type. The profit loading is $\lambda=3 \%$ of the pure risk premium. We have

$$
\sigma(\tilde{S}) \simeq \sqrt{10^{-3}\left(10^{5}+10^{7}\right)}=100.5, \quad \ell=6.0, \quad r=0.060
$$


According to the above theorem, the reinsurance arrangement which maximizes the underwriting risk return ratio is a surplus treaty with a retention of 1 . On a net basis we have

$$
\sigma\left(\tilde{S}_{\text {net }}\right) \simeq \sqrt{10^{-3} \cdot\left(10^{5}+10^{3}\right)}=10.05, \quad \ell=3.03, \quad r=0.301
$$

The net underwriting risk return ratio is much higher than the gross.

\subsection{Catastrophe Exposure}

Let $\tilde{S}=\sum_{i=1}^{n} \tilde{X}_{i}$ be a portfolio of individual risks where each risk is the sum of an ordinary risk and of a catastrophe risk:

$$
\tilde{X}_{i}={ }_{o} \tilde{X}_{i}+{ }_{c} \tilde{X}_{i}
$$

We have thus

$$
\tilde{S}=\sum_{i=1}^{n}{ }_{o} \tilde{X}_{i}+\sum_{i=1}^{n}{ }_{c} \tilde{X}_{i}
$$

It is further assumed that

$$
\operatorname{Cov}\left({ }_{o} \tilde{X}_{i},{ }_{0} \tilde{X}_{j}\right)=\delta_{i j} \sigma_{0}^{2} \quad \text { for all } i, j, \text { where } \delta_{i j}= \begin{cases}1 & i=j \\ 0 & i \neq j\end{cases}
$$

and that

$$
\operatorname{Cov}\left({ }_{c} \tilde{X}_{i}, \tilde{X}_{j}\right)=\sigma_{c}^{2} \text { for all } i, j
$$

i.e. ordinary risks are uncorrelated and catastrophe risks are perfectly correlated. It is further assumed that

$$
\operatorname{Cov}\left({ }_{o} \tilde{X}_{i}, \tilde{X}_{j}\right)=0 \text { for all } i, j \text {. }
$$

It follows that

$$
\operatorname{Cov}\left(\tilde{X}_{i}, \tilde{X}_{j}\right)=\operatorname{Cov}\left({ }_{o} \tilde{X}_{i}+{ }_{c} \tilde{X}_{i},{ }_{o} \tilde{X}_{j}+{ }_{c} \tilde{X}_{j}\right)=\delta_{i j} \sigma_{0}^{2}+\sigma_{c}^{2}
$$

and

$$
\operatorname{Var}(\tilde{S})=n \sigma_{0}^{2}+n^{2} \sigma_{c}^{2}
$$

Let us now assume that the catastrophe exposure is reinsured through a per event excess of loss reinsurance with retention $x$

$$
S_{\text {net }}=\sum_{i=1}^{n}{ }_{0} \tilde{X}_{i}+\left(\sum_{i=1}^{n}{ }_{c} \tilde{X}_{i}\right) \wedge x
$$

where $x \wedge y$ denotes the minimum of $x$ and $y$. 
To compute the value of

$$
\left(\sum_{i=1}^{n}{ }_{c} \tilde{X}_{i}\right) \wedge x
$$

as a function of $x$ we would need to make distributional assumptions on the catastrophe risk. We make the extreme assumption that the catastrophe risk is fully reinsured, i.e. $x=0$.

As a consequence we have

$$
\operatorname{Var}\left(\tilde{S}_{\text {net }}\right)=n \sigma_{0}^{2} .
$$

Let $\mu_{o}$ and $\mu_{c}$ denote the pure risk premium of an ordinary risk and of a catastrophe risk respectively. Let $\lambda_{o}$ and $\lambda_{c}$ denote the premium loading of an ordinary risk and of a catastrophe risk respectively. We have

$$
r=\frac{\ell}{\sigma(\tilde{S})}=\frac{n\left(\mu_{0} \lambda_{0}+\mu_{c} \lambda_{c}\right)}{\left(n \sigma_{0}^{2}+n^{2} \sigma_{c}^{2}\right)^{\frac{1}{2}}}=\frac{\mu_{0} \lambda_{0}+\mu_{c} \lambda_{c}}{\left(\frac{\sigma_{0}^{2}}{n}+\sigma_{c}\right)^{\frac{1}{2}}}
$$

Assuming that the loading of the reinsurance premium for the catastrophe risk is the same loading as for the original catastrophe risk, we obtain

$$
r_{n e t}=\sqrt{n} \frac{\mu_{0} \lambda_{0}}{\sigma_{0}}
$$

which is usually much larger than $r$.

\section{Numerical Example}

$$
\begin{aligned}
{ }_{0} \tilde{X}_{i} & =\left\{\begin{array}{rlc}
100 & \text { with probability } & 10^{-3} \\
0 & \text { with probability } & 0.999
\end{array}\right. \\
{ }_{c} \tilde{X}_{i} & =\left\{\begin{array}{lll}
5 & \text { with probability } & 10^{-3} \\
0 & \text { with probability } & 0.99
\end{array}\right.
\end{aligned}
$$

${ }_{0} \tilde{X}_{i}$ could be a fire claim and ${ }_{c} \tilde{X}_{i}$ an earthquake claims from a given fire policy.

We have

$$
\mu_{0}=0.1, \mu_{c}=0.05, \sigma_{0} \simeq 10^{-\frac{3}{2}} \cdot 100=3.16, \sigma_{c} \simeq 10^{-1} \cdot 5=0.5
$$

Let us assume that

$$
\lambda_{0}=5 \%, \lambda_{c}=20 \% \text { and } n=10^{5} .
$$


We obtain

$$
\begin{aligned}
& \sigma(\tilde{S})=50^{\prime} 010 \quad \ell=1^{\prime} 500 \quad r=0.030 \\
& \sigma\left(\tilde{S}_{n e t}\right)=1^{\prime} 000 \quad \ell_{n e t}=500 \quad r_{n e t}=0.500
\end{aligned}
$$

The net underwriting risk return ratio is much higher than the gross. Assuming $\tau=0.25$ we obtain the following amount of required equity

$$
u=\tau^{-1} \frac{\sigma^{2}\left(\tilde{S}_{n e t}\right)}{\ell_{n e t}}=8^{\prime} 000
$$

which leads to the following optimal risk, excess return pair

$$
\mu=\frac{\ell_{\text {net }}}{u}=6.25 \%, \quad \sigma=\frac{\sigma\left(\tilde{S}_{n e t}\right)}{u}=12.5 \% .
$$

\subsection{Portfolio Diversification}

Let $\tilde{X}_{1}, \tilde{X}_{2}, \ldots, \tilde{X}_{n}$ denote the different insurance portfolios of our company (e.g. homeowners, private automobile, commercial multiperil, commercial automobile, assumed reinsurance business, etc.).

Let

$$
\pi\left(\tilde{X}_{i}\right)=E\left(\tilde{X}_{i}\right)+\ell_{i}
$$

denote the premium of portfolio $\tilde{X}_{i}, \ell_{i}$ is thus the corresponding loading.

We use the following notation

$$
\sigma_{i j}=\operatorname{Cov}\left(\tilde{X}_{i}, \tilde{X}_{j}\right) \quad \sum=\left(\sigma_{i j}\right)
$$

We assume that the company keeps a share $\alpha_{i}$ of portfolio $\tilde{X}_{i}$ for own account and cedes a share $\left(1-\alpha_{i}\right)$ to its reinsurers.

The combined net portfolio of the company is thus

$$
\tilde{S}_{n e t}=\alpha_{1} \tilde{X}_{1}+\alpha_{2} \tilde{X}_{2}+\ldots+\alpha_{n} \tilde{X}_{n}
$$

and its combined net profit loading is

$$
\ell_{n e t}=\alpha_{1} \ell_{1}+\alpha_{2} \ell_{2}+\ldots+\alpha_{n} \ell_{n}
$$

\section{Theorem}

We assume that $\sum^{-1}$ exists.

1. The vector $\underline{\alpha}^{\prime}=\left(\alpha_{1}, \alpha_{2}, \ldots, \alpha_{n}\right)$ which maximizes the net underwriting risk return ratio

$$
r_{n e t}=\frac{\ell_{n e t}}{\sigma\left(\tilde{S}_{n e t}\right)}
$$


is given by

$$
\underline{\alpha}=c \cdot \Sigma^{-1} \cdot \underline{\ell}
$$

where $\underline{\ell}^{\prime}=\left(\ell_{1}, \ell_{2}, \ldots, \ell_{n}\right)$ and $c$ is a scalar which is chosen in such a way that $\max _{i=1, \ldots, n} \alpha_{i}=1$.

The optimal risk return ratio is equal to

$$
r_{n e t}=\left(\underline{\ell}^{\prime} \Sigma^{-1} \underline{\ell}\right)^{\frac{1}{2}}
$$

2. $\underline{\alpha}$ maximizes the risk return ratio if and only if the net loadings $\left(\alpha_{i} \ell_{i} \quad i=1, \ldots, n\right)$ are equal to the fair loadings.

\section{Remark}

The solution $\underline{\alpha}$ provided by the theorem is only meaningful if $\alpha_{i} \geq 0$ for all $i$. It is indeed unrealistic to assume that the company can take a short position in any of the insurance portfolios $\tilde{X}_{i}$. To find a solution $\underline{\alpha}$ which always satisfies the condition $\underline{\alpha} \geq 0$ is a convex optimization problem with restrictions. It is a standard problem in finance theory, see for instance W.F. Sharpe (1970).

\section{Proof}

1. We have to maximize the following expression

$$
r=\frac{\alpha_{1} \ell_{1}+\alpha_{2} \ell_{2}+\ldots+\alpha_{n} \ell_{n}}{\left(\sum_{i, j} \alpha_{i} \alpha_{j} \sigma_{i j}\right)^{\frac{1}{2}}}
$$

deriving with respect to $\alpha_{1}, \alpha_{2}, \ldots, \alpha_{n}$ and equating the expression to 0 , we obtain

$$
\begin{aligned}
& \frac{\delta r}{\delta \alpha_{1}}=\frac{\ell_{1} \sigma\left(\tilde{S}_{n e t}\right)-\ell_{n e t \frac{1}{2} \sigma\left(\tilde{S}_{n e t}\right)^{-1}}\left(2 \sum_{j=1}^{n} \alpha_{j} \sigma_{1 j}\right)}{\sigma^{2}\left(\tilde{S}_{n e t}\right)}=0 \\
& \vdots \\
& \vdots \\
& \frac{\partial r}{\delta \alpha_{n}}=\frac{\ell_{n} \sigma\left(\tilde{S}_{n e t}\right)-\ell_{n e t} \frac{1}{2} \sigma\left(\tilde{S}_{n e t}\right)^{-1}\left(2 \sum_{j=1}^{n} \alpha_{j} \sigma_{n j}\right)}{\sigma^{2}\left(\tilde{S}_{n e t}\right)}=0
\end{aligned}
$$


and after some straightforward rearrangement of terms

$$
\begin{gathered}
\ell_{1} \sigma^{2}\left(\tilde{S}_{n e t}\right)=\ell_{n e t_{j}} \sum_{j=1}^{n} \alpha_{j} \sigma_{1 j} \\
\vdots \\
\vdots \\
\ell_{n} \sigma^{2}\left(\tilde{S}_{n e t}\right)=\ell_{n e t_{j}} \sum_{j=1}^{n} \alpha_{j} \sigma_{n j}
\end{gathered}
$$

or in matrix notation

$$
\begin{gathered}
\underline{\ell} \frac{\sigma^{2}\left(\tilde{S}_{n e l}\right)}{\ell_{\text {nel }}}=\Sigma \underline{\alpha} \\
\underline{\alpha}=c \Sigma^{-1} \underline{\ell}
\end{gathered}
$$

This proves the first part of the theorem. (Note that by definition $\underline{\alpha}$ is only defined up to a norming constant $c$.)

We now prove the statement about $r_{n e t}$.

$$
\begin{gathered}
\operatorname{Var}(\tilde{S})=\underline{\alpha}^{\prime} \Sigma \underline{\alpha}=c^{2} \underline{\ell}^{\prime} \Sigma^{-1} \Sigma \Sigma^{-1} \underline{\ell}=\left(c \underline{\ell}^{\prime}\right)\left(c \Sigma^{-1} \underline{\ell}\right)=c \underline{\ell}^{\prime} \underline{\alpha} \\
r_{\text {net }}=\frac{\underline{\alpha^{\prime}} \underline{\ell}}{\sqrt{c}\left(\underline{\alpha}^{\prime} \underline{\ell}\right)^{\frac{1}{2}}}=\frac{1}{\sqrt{c}}\left(\underline{\alpha}^{\prime} \underline{\ell}\right)^{\frac{1}{2}}=\frac{\sqrt{c}}{\sqrt{c}}\left(\underline{\ell}^{\prime} \Sigma^{-1} \underline{\ell}\right)^{\frac{1}{2}} \\
r_{\text {net }}=\left(\underline{\ell}^{\prime} \Sigma^{-1} \underline{\ell}\right)^{\frac{1}{2}}
\end{gathered}
$$

2. $\alpha_{i} \ell_{i} i=1, \ldots, n$ are the fair loadings if and only if $\alpha_{i} \ell_{i}=c \cdot \operatorname{Cov}\left(\alpha_{i} \tilde{X}_{i}, \tilde{S}_{n e t}\right) \quad i=1, \ldots, n$ for some constant $c$. This in turn is equivalent with the following system of equations

$$
\begin{gathered}
\alpha_{i} \ell_{i}=c \cdot \sum_{j=1}^{n} \alpha_{i} \alpha_{j} \sigma_{i j} \quad i=1,2, \ldots, n \\
\ell_{i}=c \cdot \sum_{j=1}^{n} \sigma_{i j} \alpha_{j} \quad i=1,2, \ldots, n \\
\underline{\ell}=c \Sigma \underline{\alpha} \\
\underline{\alpha}=c^{-1} \Sigma^{-1} \underline{\ell}
\end{gathered}
$$

which is equivalent with $\underline{\alpha}$ maximizing the risk return ratio.

q.e.d.

\section{Numerical Example}

There are three portfolios with

$$
\begin{array}{lll}
\sigma_{11}=1 & \ell_{1}=0.2 & \Rightarrow \frac{\ell_{1}}{\sqrt{\sigma_{11}}}=20 \% \\
\sigma_{22}=4 & \ell_{2}=0.2 & \Rightarrow \frac{\ell_{2}}{\sqrt{\sigma_{22}}}=30 \%
\end{array}
$$


We think of $\tilde{X}_{1}$ and $\tilde{X}_{2}$ as of a motor portfolio and a homeowners portfolio respectively. We assume that both portfolios are exposed to the same natural peril (e.g. storm), which is only reinsured in excess of a substantial retention. The correlation between the two portfolios is therefore positive. Let us assume that it is equal to 0.20 .

The third class of business consists of industrial risks with

$$
\sigma_{33}=9 \cdot(1.5)^{2}=20.25 \quad \ell_{3}=1.8 \quad \Rightarrow \frac{\ell_{3}}{\sqrt{\sigma_{33}}}=40 \%
$$

The interpretation is that for the same premium income as the homeowners portfolio, the industrial portfolio has a standard deviation of 3 , instead of 2 for the homeowners portfolio. The industrial portfolio has $50 \%$ more volume than the homeowners portfolio. It is assumed that the industrial portfolio and each of the personal lines portfolio are uncorrelated. We have thus

$$
\sum=\left(\begin{array}{lll}
1 & 0.4 & 0 \\
0.4 & 4 & 0 \\
0 & 0 & 20.25
\end{array}\right), \quad \underline{\ell}=\left(\begin{array}{l}
0.2 \\
0.6 \\
1.8
\end{array}\right)
$$

From our theorem we obtain that the optimal retentions are

$$
\underline{\alpha}^{\prime}=(1, \quad 0.93, \quad 0.61)
$$

yielding

$$
\sigma\left(\tilde{S}_{n e t}\right)=3.57 \quad \ell_{n e t}=1.85 \quad r_{n e t}=0.518
$$

Thus the optimal risk return ratio is much higher than each of the risk return ratios of the individual classes.

Let $\tilde{S}$ be the gross combined portfolio $\tilde{S}=\tilde{X}_{1}+\tilde{X}_{2}+\tilde{X}_{3}$ we have

$$
\sigma(\tilde{S})=5.10 \quad \ell=2.60 \quad r=\frac{\ell_{1}+\ell_{2}+\ell_{3}}{\left(\sum_{i, j} \sigma_{i j}\right)^{\frac{1}{2}}}=\frac{2.6}{(26.05)^{\frac{1}{2}}}=0.509
$$

which is nearly as high as the optimal risk return ratio. To achieve the optimal ratio the company must cede $7 \%$ of its homeowners business and $39 \%$ of its industrial business. It must thus forgo an expected profit of 0.75 out of a total expected profit of 2.6. It is questionable whether in this case the slight improvement in the risk return ratio is worth this sacrifice.

Let us assume that for given $R=E(\tilde{\Delta} u)$ and $V=\operatorname{Var}(\tilde{\Delta} u)$ the company chooses the amount of equity $u$ in such a way as to maximize

$$
2 \tau \frac{R}{u}-\frac{V}{u^{2}} \text {. }
$$


This is tantamount to utilizing Markowitz's objective function to determine the optimal amount of capital for a given risk and return. The optimal amount of equity is

$$
u=\tau^{-1} \frac{V}{R}
$$

For $\tau=0.25$ and utilizing the notation

$$
\mu=E(\tilde{\delta}(u)), \quad \sigma=\operatorname{Var}^{\frac{1}{2}}(\tilde{\delta}(u))
$$

we obtain

\begin{tabular}{lllll}
\hline $\begin{array}{c}\text { Portfolio } \\
\text { number }\end{array}$ & $\boldsymbol{r}$ & $\boldsymbol{\sigma}$ & $\boldsymbol{\mu}$ & $\boldsymbol{u}$ \\
\hline 1 & 0.200 & $5 \%$ & $1 \%$ & 20.0 \\
2 & 0.300 & $7.5 \%$ & $2.25 \%$ & 26.67 \\
3 & 0.400 & $10 \%$ & $4 \%$ & 45.0 \\
4 & 0.509 & $12.75 \%$ & $6.5 \%$ & 40.0 \\
5 & 0.518 & $12.95 \%$ & $6.71 \%$ & 27.56 \\
\hline
\end{tabular}

where portfolio number 4 is the combined portfolio and portfolio number 5 is the optimal portfolio.

This example illustrates that combining portfolios results in substantial capital savings and improvements of the risk return ratio. This example also illustrates the fact that, when we combine portfolios in a non optimal way, there is a cross subsidization between portfolios: Let $\tilde{S}$ denote the gross combined portfolio. The fair loadings are

$$
\ell_{i}=\mu \cdot u \cdot \frac{\operatorname{Cov}\left(\tilde{X}_{i}, \tilde{S}\right)}{\operatorname{Var}(\tilde{S})}
$$

thus

$$
\ell_{1}=6.5 \% \cdot 40.0 \cdot \frac{1.4}{26.05}=0.14 \quad \ell_{2}=0.44 \quad \ell_{3}=2.02
$$

whereas the actual loadings are

$$
\ell_{1}=0.20 \quad \ell_{2}=0.60 \quad \ell_{3}=1.80
$$

There is a subsidization of $\tilde{X}_{3}$ from $\tilde{X}_{1}$ and $\tilde{X}_{2}$. 


\section{LOSS RESERVE RISK}

\subsection{Individual Accident Year}

Since we only consider one accident year, we can assume that the development year $t$ of risk $\tilde{X}$ is also the financial year $t$ of the company. This amounts to a renumbering of the financial years. We first analyze the problem on an undiscounted basis. Later we introduce discounting.

Let $\tilde{X}$ denote a risk, or a portfolio of risks pertaining to a given accident year. Let $\pi(\tilde{X})$ and $\ell$ denote respectively the premium and the loading of risk $\tilde{X}$. We have

$$
\pi(\tilde{X})=E(\tilde{X})+\ell .
$$

As with all other random variables we assume that $E\left(\tilde{X}^{2}\right)$ is finite. Let us assume that $\tilde{X}$ is paid out over $\omega$ development years.

$$
\tilde{X}=\sum_{t=1}^{\omega} \tilde{P}_{t}
$$

$\tilde{P}_{t}$ denotes the payment made in development year $t$ in respect of risk $\tilde{X}$. Let $\mathcal{H}_{t}$ denote the information of the company on risk $\tilde{X}$ in development year $t . \mathcal{H}_{0}$ is the information on the risk prior to underwriting it and we have thus $E(\tilde{X})=E\left(\tilde{X} \mid \mathcal{H}_{0}\right)$.

We further introduce the following notation

$$
\tilde{X}_{t}=E\left(\tilde{X} \mid \mathcal{H}_{t}\right)
$$

$\tilde{X}_{t}$ is the company's estimate of risk $\tilde{X}$ in development year $t$.

We assume that $\mathcal{H}_{0}, \mathcal{H}_{1}, \ldots, \mathcal{H}_{t}, \ldots$ is an increasing sequence of $\sigma$-algebras. It is easily seen that $\tilde{X}_{t}$ is a martingale. Let

$$
\tilde{L}_{t}=E\left(\tilde{P}_{t+1}+\tilde{P}_{t+2}+\ldots \mid \mathcal{H}_{t}\right)
$$

be the loss reserve of the company at the end of development year $t$ in respect of risk $\tilde{X}$.

Based on the pure risk premium $E(\tilde{X})$, the contribution to results produced by risk $\tilde{X}$ in the successive development years are as follows

$$
\tilde{R}_{t}=\tilde{L}_{t-1}-\tilde{P}_{t}-\tilde{L}_{t} \quad t=1,2, \ldots
$$

and the following relation holds true

$$
\tilde{R}_{t}=E\left(\tilde{X} \mid \mathcal{H}_{t-1}\right)-E\left(\tilde{X} \mid \mathcal{H}_{t}\right) \quad t=1,2, \ldots
$$

$\tilde{R}_{t}$ is the difference process of a martingale (i.e. of $E\left(-\tilde{X} \mid \mathcal{H}_{t}\right)$ ). 
Note that according to our terminology, $\tilde{R}_{t}$ is the underwriting risk and $\tilde{R}_{2}+\ldots+\tilde{R}_{\omega}$ is the loss reserve risk.

Since $-\tilde{X}_{t}=-E\left(\tilde{X} \mid \mathcal{H}_{t}\right)$ is a martingale and $\tilde{R}_{t}$ is the corresponding difference process, the following holds true

$$
\begin{gathered}
E\left(\tilde{R}_{t}\right)=0 \quad t=1,2, \ldots, \quad \operatorname{Cov}\left(\tilde{R}_{t}, \tilde{R}_{s}\right)=0 \quad t \neq s, \\
\operatorname{Var}(\tilde{X})=\sum_{t=1}^{\omega} \operatorname{Var}\left(\tilde{R}_{t}\right) .
\end{gathered}
$$

Let $\ell$ denote the loading for profit pertaining to risk $\tilde{X}$. We make the assumption that $\ell$ is earned over the whole development period of risk $\tilde{X}$. The amount earned during development year $t$ is

$$
\ell_{t}=\ell \cdot \frac{\operatorname{Var}\left(\tilde{R}_{t}\right)}{\operatorname{Var}(\tilde{X})}
$$

The above ensures that $\sum_{t=1}^{\omega} \ell_{t}=\ell$.

We now introduce discounting. Let $\tilde{\delta}(u)$, a random variable, denote the interest rate intensity at time $u$. The present value at time $s$ of one monetary unit paid at time $t$ is then

$$
\tilde{v}(s, t)=e^{-\int_{s}^{t} \tilde{\delta}(u) d u}
$$

Let $\mathcal{G}_{t}$ denote the cumulative information on the interest rate intensity up to the end of financial year $t$ (which is also development year $t$ of risk $X$ ). It is assumed that $\mathcal{G}_{0}, \mathcal{G}_{1}, \ldots, \mathcal{G}_{t}, \ldots$ is an increasing sequence of $\sigma$-algebras.

We have now

$$
\tilde{X}=\tilde{v}(0,1) \cdot \tilde{P}_{1}+\tilde{v}(0,2) \cdot \tilde{P}_{2}+\ldots+\tilde{v}(0, \omega) \cdot \tilde{P}_{\omega}
$$

Let

$$
\tilde{L}_{t}=E\left(\sum_{s=1}^{\omega-t} \tilde{v}(t, t+s) \tilde{P}_{t+s} \mid \mathcal{H}_{t}, \mathcal{G}_{t}\right)
$$

be the loss reserve of the company in respect of risk $\tilde{X}$ at the end of development year $t$. As a special case we have $L_{0}=E(\tilde{X})$. 
The loss development risk in development year $t$ is

$$
\begin{aligned}
\tilde{R}_{t}= & \tilde{L}_{t-1}-\tilde{P}_{t}-\tilde{L}_{t} \\
\tilde{R}_{t}= & E\left(\sum_{s=1}^{\omega-t+1} \tilde{v}(t-1, t-1+s) \tilde{P}_{t-1+s} \mid \mathcal{H}_{t-1}, \mathcal{G}_{t-1}\right)-\tilde{P}_{t} \\
& -E\left(\sum_{s=1}^{\omega-t} \tilde{v}(t, t+s) \tilde{P}_{t+s} \mid \mathcal{H}_{t}, \mathcal{G}_{t}\right) \\
= & {\left[E\left(\sum_{s=0}^{\omega-t} \tilde{v}(t-1, t+s) \tilde{P}_{t+s} \mid \mathcal{H}_{t-1}, \mathcal{G}_{t-1}\right)\right.} \\
& \left.-E\left(\sum_{s=0}^{\omega-t} \tilde{v}(t-1, t+s) \tilde{P}_{t+s} \mid \mathcal{H}_{t}, \mathcal{G}_{t-1}\right)\right] \\
& +\left[E\left(\sum_{s=0}^{\omega-t} \tilde{v}(t-1, t+s) \tilde{P}_{t+s} \mid \mathcal{H}_{t}, \mathcal{G}_{t-1}\right)\right. \\
& \left.-E\left(\sum_{s=0}^{\omega-t} \tilde{v}(t, t+s) \tilde{P}_{t+s} \mid \mathcal{H}_{t}, \mathcal{G}_{t}\right)\right] \\
\tilde{R}_{t}= & { }_{1} \tilde{R}_{t}+{ }_{2} \tilde{R}_{t}
\end{aligned}
$$

\section{Assumption 6}

The interest rate process and the claims process are stochastically independent.

Under the above assumption we obtain

$$
{ }_{1} \tilde{R}_{t}=\sum_{s=0}^{\omega-t} E\left(\tilde{v}(t-1, t+s) \mid \mathcal{G}_{t-1}\right) \cdot\left(E\left(\tilde{P}_{t+s} \mid \mathcal{H}_{t-1}\right)-E\left(\tilde{P}_{t+s} \mid \mathcal{H}_{t}\right)\right)
$$

${ }_{1} \tilde{R}_{t}$ is the loss reserve development risk. It is seen at once that $E\left({ }_{1} \tilde{R}_{t}\right)=0$. In addition the company will earn a profit loading $\ell_{t}$ as defined above, for assuming the risk ${ }_{1} \tilde{R}_{t}$. 
We also have

$$
\begin{aligned}
{ }_{2} \tilde{R}_{t}= & \sum_{s=0}^{\omega-t} E\left(\tilde{P}_{t+s} \mid \mathcal{H}_{t}\right) \cdot\left(E\left(\tilde{v}(t-1, t+s) \mid \mathcal{G}_{t-1}\right)-E\left(\tilde{v}(t, t+s) \mid \mathcal{G}_{t}\right)\right) \\
{ }_{2} \tilde{R}_{t}= & \sum_{s=0}^{\omega-t} E\left(\tilde{P}_{t+s} \mid \mathcal{H}_{t}\right) \cdot\left(E\left(\tilde{v}(t-1, t+s) \mid \mathcal{G}_{t-1}\right)-E\left(\tilde{v}(t-1, t+s) \mid \mathcal{G}_{t}\right)\right. \\
& \left.+E\left(\tilde{v}(t-1, t+s) \mid \mathcal{G}_{t}\right)-E\left(\tilde{v}(t, t+s) \mid \mathcal{G}_{t}\right)\right) \\
{ }_{2} \tilde{R}_{t}= & \sum_{s=0}^{\omega-t} E\left(\tilde{P}_{t+s} \mid \mathcal{H}_{t}\right) \cdot\left(E\left(\tilde{v}(t-1, t+s) \mid \mathcal{G}_{t-1}\right)-E\left(\tilde{v}(t-1, t+s) \mid \mathcal{G}_{t}\right)\right) \\
& +\sum_{s=0}^{\omega-t} E\left(\tilde{P}_{t+s} \mid \mathcal{H}_{t}\right) \cdot E\left(\tilde{v}(t-1, t+s) \mid \mathcal{G}_{t}\right) \cdot\left(1-\tilde{v}^{-1}(t-1, t)\right)
\end{aligned}
$$

and it is seen that the first term is the yield curve risk stemming from the discounting of the loss reserves and the second term is the unwinding of the discount.

$-{ }_{2} \tilde{R}_{t}$ can be viewed as the yield in financial year $t$ of a bond portfolio with the amounts $E\left(\tilde{P}_{t} \mid \mathcal{H}_{t}\right), E\left(\tilde{P}_{t+1} \mid \mathcal{H}_{t}\right), \ldots, E\left(\tilde{P}_{\omega} \mid \mathcal{H}_{t}\right)$ maturing at time $t, t+1, \ldots, \omega$ respectively. The risk ${ }_{2} \tilde{R}_{t}$ can therefore be perfectly hedged through asset liability matching.

\subsection{Different Accident Years}

Let $\tilde{X}_{1}, \tilde{X}_{2}, \ldots, \tilde{X}_{\omega}$ denote a risk or a portfolio of risks pertaining to accident years $1,2, \ldots, \omega$. Let $\tilde{P}_{t, s}$ denote the claims payment made in respect of accident year $t$, in development year $s$. It is assumed that each $\tilde{X}_{t}$ is paid over $\omega$ development years. We have

$$
\tilde{X}_{t}=\sum_{s=1}^{\omega-t+1} \tilde{v}(t-1, t-1+s) P_{t, s}
$$

where $v(s, t)$ is defined as the preceding subsection. $\mathcal{H}_{t, s}(s=1,2, \ldots, \omega)$ is the $\sigma$-algebra generated by $\left\{\tilde{P}_{t, 1}, \tilde{P}_{t, 2}, \ldots, \tilde{P}_{t, s}\right\} . \mathcal{G}_{t}$ is the $\sigma$-algebra generated by $\{\tilde{\delta}(u) \mid u \leq t\}$. The loss reserve held by the company in respect of accident year $t$ at the beginning of financial year $\omega$ is

$$
L_{t, \omega-t}=E\left(\sum_{s=\omega-t+1}^{\omega} \tilde{v}(\omega-1, s+t-1) \tilde{P}_{t, s} \mid \mathcal{H}_{t, \omega-t}, \mathcal{G}_{\omega-1}\right) .
$$

At the end of financial year $\omega$ it pays $P_{t, \omega-t+1}$ and puts up a reserve

$$
L_{t, \omega-t+1}=E\left(\sum_{s=\omega-t+2}^{\omega} \tilde{v}(\omega, s+t-1) \tilde{P}_{t, s} \mid \mathcal{H}_{t, \omega-t+1}, \mathcal{G}_{\omega}\right) .
$$


The risk materializing during financial year $\omega$ in respect of accident year $t$ is

$$
\tilde{R}_{t, \omega-t+1}=L_{t, \omega-t}-P_{t, \omega-t+1}-L_{t, \omega-t+1} .
$$

And the overall loss reserve risk is thus

$$
\tilde{\Delta} L=-\sum_{t=1}^{\omega-1} R_{t, \omega-t+1} .
$$

Note that $\tilde{R}_{\omega, 1}$ is the underwriting risk in respect of accident year $\omega$ and is therefore not part of the loss reserve risk.

Upon rearranging terms, we obtain

$$
\begin{aligned}
\tilde{R}_{t, \omega-t+1}= & E\left(\sum_{s=\omega-t+1}^{\omega} \tilde{v}(\omega-1, s+t-1) \tilde{P}_{t, s} \mid \mathcal{H}_{t, \omega-t}, \mathcal{G}_{\omega-1}\right) \\
& -E\left(\sum_{s=\omega-t+1}^{\omega} \tilde{v}(\omega, s+t-1) \tilde{P}_{t, s} \mid \mathcal{H}_{t, \omega-t+1}, \mathcal{G}_{\omega}\right) \\
= & {\left[E\left(\sum_{s=\omega-t+1}^{\omega} \tilde{v}(\omega-1, s+t-1) \tilde{P}_{t, s} \mid \mathcal{H}_{t, \omega-t}, \mathcal{G}_{\omega-1}\right)\right.} \\
& \left.-E\left(\sum_{s=\omega-t+1}^{\omega} \tilde{v}(\omega-1, s+t-1) \tilde{P}_{t, s} \mid \mathcal{H}_{t, \omega-t+1}, \mathcal{G}_{\omega-1}\right)\right] \\
& +\left[E\left(\sum_{s=\omega-t+1}^{\omega} \tilde{v}(\omega-1, s+t-1) \tilde{P}_{t, s} \mid \mathcal{H}_{t, \omega-t+1}, \mathcal{G}_{\omega-1}\right)\right. \\
& \left.-E\left(\sum_{s=\omega-t+1}^{\omega} \tilde{v}(\omega, s+t-1) \tilde{P}_{t, s} \mid \mathcal{H}_{t, \omega-t+1}, \mathcal{G}_{\omega}\right)\right] \\
R_{t, \omega-t+1}= & R_{t, \omega-t+1}+{ }_{2} R_{t, \omega-t+1}
\end{aligned}
$$

Using assumption 6 we obtain

$$
\begin{aligned}
{ }_{1} R_{t, \omega-t+1}= & \sum_{s=\omega-t+1}^{\omega} E\left(\tilde{v}(\omega-1, s+t-1) \mid \mathcal{G}_{\omega-1}\right) \\
& \cdot\left(E\left(\tilde{P}_{t, s} \mid \mathcal{H}_{t, \omega-t}\right)-E\left(\tilde{P}_{t, s} \mid \mathcal{H}_{t, \omega-t+1}\right)\right) \\
{ }_{2} R_{t, \omega-t+1}= & \sum_{s=\omega-t+1}^{\omega} E\left(\tilde{P}_{t, s} \mid \mathcal{H}_{t, \omega-t+1}\right) \\
& \cdot\left(E\left(\tilde{v}(\omega-1, s+t-1) \mid \mathcal{G}_{\omega-1}\right)-E\left(\tilde{v}(\omega, s+t-1) \mid \mathcal{G}_{\omega}\right)\right)
\end{aligned}
$$

Let $\tilde{\Delta} L=\tilde{\Delta} L_{1}+\tilde{\Delta} L_{2}$ with $\tilde{\Delta} L_{i}=\sum_{t=1}^{\omega-1}{ }_{i} R_{t, \omega-t+1} \quad i=1,2$. 
$\tilde{\Delta} L_{1}$ is the loss reserve development risk and $\tilde{\Delta} L_{2}$ is the yield curve risk combined with the unwinding of the discount.

It is easily seen that $E\left(\tilde{\Delta} L_{1}\right)=0$. In return for the assumption of the risk $\tilde{\Delta} L_{1}$ the company earns a profit loading

$$
\ell_{1}=\sum_{t=1}^{\omega-1} \ell_{t, \omega-t+1}
$$

where $\ell_{t, \omega-t+1}$ is the profit loading pertaining to accident year $t$ in development year $\omega-t+1$ (see section 3.1).

Upon rearranging terms we obtain

$$
\begin{aligned}
\tilde{\Delta} L_{2}= & -\sum_{t=1}^{\omega-1} R_{t, \omega-t+1} \\
\tilde{\Delta} L_{2}= & \sum_{t=1}^{\omega-1} \sum_{s=\omega-t+1}^{\omega} E\left(\tilde{\boldsymbol{P}}_{t, s} \mid \mathcal{H}_{t, \omega-t+1}\right) \cdot\left(E\left(\tilde{v}(\omega, s+t-1) \mid \mathcal{G}_{\omega}\right)\right. \\
& \left.-E\left(\tilde{v}(\omega-1, s+1-1) \mid \mathcal{G}_{\omega-1}\right)\right) \\
\tilde{\Delta} L_{2}= & \sum_{s=0}^{\omega-2} k_{s}\left(E\left(\tilde{v}(\omega, \omega+s) \mid \mathcal{G}_{\omega}\right)-E\left(\tilde{v}(\omega-1, \omega+s) \mid \mathcal{G}_{\omega-1}\right)\right)
\end{aligned}
$$

with

$$
k_{s}=\sum_{t=s+1}^{\omega} E\left(\tilde{P}_{t, \omega+s+1-t} \mid \mathcal{H}_{t, \omega-t+1}\right) .
$$

Thus

$$
\begin{aligned}
\tilde{\Delta} L_{2}= & \sum_{s=0}^{\omega-2} k_{s}\left(E\left(\tilde{v}(\omega, \omega+s) \mid \mathcal{G}_{\omega}\right)-E\left(\tilde{v}(\omega-1, \omega+s) \mid \mathcal{G}_{\omega}\right)\right. \\
& \left.+E\left(\tilde{v}(\omega-1, \omega+s) \mid \mathcal{G}_{\omega}\right)-E\left(\tilde{v}(\omega-1, \omega+s) \mid \mathcal{G}_{\omega-1}\right)\right) \\
\tilde{\Delta} L_{2}= & \sum_{s=0}^{\omega-2} k_{s}\left(E\left(\tilde{v}(\omega-1, \omega+s) \mid \mathcal{G}_{\omega}\right) \cdot\left(\tilde{v}^{-1}(\omega-1, \omega)\right)\right) \\
& +\sum_{s=0}^{\omega-2} k_{s}\left(E\left(\tilde{v}(\omega-1, \omega+s) \mid \mathcal{G}_{\omega}\right)-E\left(\tilde{v}(\omega-1, \omega+s) \mid \mathcal{G}_{\omega-1}\right)\right)
\end{aligned}
$$

where the first term is the unwinding of the discount and the second term is the yield curve risk stemming from the discounting of the loss reserves. We have thus

$$
\tilde{\Delta} L_{2}=\tilde{R}_{L} \cdot L
$$


where $L=\sum_{s=0}^{\omega-2} k_{s} E\left(\tilde{v}(\omega-1, \omega+s) \mid \mathcal{G}_{\omega-1}\right)$ is the total discounted loss reserves at the beginning of financial year $\omega$ and $\tilde{R}_{L}$ is the yield for financial year $\omega$ of a bond portfolio with the amounts $k_{s}$ maturing at the end of financial year $\omega+s \quad(s=0,1, \ldots, \omega-2) . \tilde{R}_{L}$ is the rate of return of a bond portfolio with the same maturities as the liabilities of the company. $\Delta L_{2}$ can thus be perfectly hedged through asset liability matching.

In summary the loss reserve risk consists of two parts

$$
\tilde{\Delta} L=\left(\tilde{\Delta} L_{1}-\ell_{1}\right)+\tilde{R}_{L} \cdot L
$$

a loss reserve development risk $\left(\tilde{\Delta} L_{1}\right)$ and a yield curve risk $\left(\tilde{R}_{L} \cdot L\right)$.

\section{General Model Including Asset Risk}

\subsection{Optimality Criterion}

We have obtained the following representation for the return of the company during the financial year

$$
\tilde{\Delta} u=(E(\tilde{S})+\ell-\tilde{S})+\left(\ell_{1}-\tilde{\Delta} L_{1}\right)-\tilde{R}_{L} \cdot L+\tilde{\Delta} A
$$

The first two terms are insurance risks (underwriting and loss reserve development risk), the last two terms are financial risks (yield curve risk and asset risk).

It is assumed that there are $n$ different categories of assets. $\tilde{R}_{j}$, a random variable, denotes the return of asset category $j . A_{j}$ denotes the amount invested by the company in asset category $j$. We have

$$
\tilde{\Delta} A=\sum_{j=1}^{n} \tilde{R}_{j} \cdot A_{j} .
$$

Let $\rho_{0}$ denote the return of the risk free asset. We obtain the following representation for the excess return of the company

$$
\tilde{\Delta} u-\rho_{0} u=(E(\tilde{S})+\ell-\tilde{S})+\left(\ell_{1}-\tilde{\Delta} L_{1}\right)-\left(\tilde{R}_{L}-\rho_{0}\right) \cdot L+\sum_{j=1}^{n}\left(\tilde{R}_{j}-\rho_{0}\right) \cdot A_{j}
$$

where we have used the fact that the sum of the liabilities of the company is equal to the sum of its assets

$$
L+u=\sum_{j=1}^{n} A_{j}
$$


Let

$$
\begin{gathered}
E(\tilde{S})+\ell-\tilde{S}=\sum_{i=1}^{m} E\left(\tilde{X}_{i}\right)+\ell_{i}-\tilde{X}_{i} \\
\ell_{1}-\tilde{\Delta} L_{1}=\sum_{i=1}^{m^{\prime}} \ell_{i}^{\prime}-\tilde{X}_{i}^{\prime} \quad\left(E\left(\tilde{X}_{i}^{\prime}\right)=0\right)
\end{gathered}
$$

and

$$
\left(\tilde{R}_{L}-\rho_{0}\right) \cdot L=\sum_{i=1}^{m^{\prime}}\left(\tilde{R}_{i}^{\prime}-\rho_{0}\right) \cdot L_{i}
$$

be a split of the underwriting risk, the loss reserve development risk and the yield curve risk into individual risks (e.g. lines of business, market segments, etc.). We assume that company keeps a share $\alpha_{i},\left(\alpha_{i} \in[0,1]\right)$ of each individual underwriting risk and cedes $1-\alpha_{i}$ via quota share reinsurance. Similarly the company retains a share $\beta_{j}$ of loss reserve development risk and of the yield curve risk $j$. The excess profit of the company now reads

$$
\begin{aligned}
\tilde{\Delta} u-\rho_{0} u= & \sum_{i=1}^{m} \alpha_{i} \cdot\left(E\left(\tilde{X}_{i}\right)+\ell_{i}-\tilde{X}_{i}\right)+\sum_{j=1}^{m^{\prime}} \beta_{j} \cdot\left(\left(\ell_{j}^{\prime}-\tilde{X}_{j}^{\prime}\right)-\left(\tilde{R}_{j}^{\prime}-\rho_{0}\right) \cdot L_{j}\right) \\
& +\sum_{i=1}^{n}\left(\tilde{R}_{j}-\rho_{0}\right) \cdot A_{j}
\end{aligned}
$$

And it is seen that portfolio optimization amounts to an 'optimal' choice of the $\alpha$ 's, $\beta$ 's and $A$ 's. We now define the optimality criterion.

Let

$$
\tilde{\delta}(u)=\frac{\tilde{\Delta} u-\rho_{0} u}{u}, \quad \mu(u)=E(\tilde{\delta}(u)), \quad \sigma^{2}(u)=\operatorname{Var}(\tilde{\delta}(u))
$$

The objective of the company is to maximize

$$
2 \tau \mu(u)-\sigma^{2}(u), \quad \text { with } \quad \tau \geq 0 .
$$

(For a discussion of the above objective function see section 2.2). As in section 2.2 we have

$$
\mu(u)=\frac{R(\underline{\alpha}, \underline{\beta}, \underline{A})}{u}, \quad \sigma^{2}(u)=\frac{V(\underline{\alpha}, \underline{\beta}, \underline{A})}{u^{2}}
$$


Thus the same arguments apply and it is seen that the efficient frontier is defined by maximizing the risk return ratio (Sharpe's ratio).

$$
\frac{\mu(u)}{\sigma(u)}=\frac{L(\underline{\alpha}, \underline{\beta}, \underline{A})}{(V(\underline{\alpha}, \underline{\beta}, \underline{A}))^{\frac{1}{2}}}=r(\underline{\alpha}, \underline{\beta}, \underline{A})
$$

Hence the following

\section{Definition}

A portfolio is optimal if and only if the corresponding risk return ratio $r(\underline{\alpha}, \beta, \underline{A})$ is maximal. In addition $\underline{\alpha}$ and $\beta$ are such that the net retained insurance profit is maximized.

Usually $r(\underline{\alpha}, \beta, \underline{A})$ is maximized under certain constraints such as $\alpha_{i} \in\left[\begin{array}{ll}0,1 & 1\end{array}\right.$ and $\beta_{j} \in[0,1]$ and, if the company is not allowed to issue securities $A_{i} \geq 0$.

Once the company portfolio has been determined, the risk return ratio and the efficient border of the company are given. The company still has to choose a specific point on the efficient frontier. This choice is equivalent to the choice of the amount of capital of the company which in turn is defined by the risk tolerance $\tau$ (see section 2.2).

Let $\tilde{\Delta} u=\sum_{i=1}^{n} \tilde{Z}_{i}$ be any split of the total risk of the company into individual risks. Since the amount of capital required to assume the total risk $\tilde{\Delta} u$ is proportional to

$$
\operatorname{Var}(\tilde{\Delta} u)=\sum_{i=1}^{n} \operatorname{Cov}\left(\tilde{Z}_{i}, \tilde{\Delta} u\right)
$$

We allocate to each individual risk $\tilde{Z}_{i}$ an amount of capital $u_{i}$, which is proportional to the contribution of that risk to the overall volatility of the result of the company

Since $\sum_{i=1}^{n} u_{i}=u$, we obtain

$$
u_{i}=k \cdot \operatorname{Cov}\left(\tilde{Z}_{i}, \tilde{\Delta} u\right) .
$$

$$
u_{i}=u \cdot \frac{\operatorname{Cov}\left(\tilde{Z}_{i}, \tilde{\Delta} u\right)}{\operatorname{Var}(\tilde{\Delta} u)}
$$

The excess profit which the company expects to achieve for assuming the risk $\sigma^{2}(\tilde{\Delta} u)$ is $\left(\rho-\rho_{0}\right) \cdot u$. It is fair to split the excess profit proportionally to the allocated capital. Thus

\section{Definition}

The fair loading of risk $\tilde{Z}_{i}$ is

$$
\left(\rho-\rho_{0}\right) \cdot u_{i}=\left(\rho-\rho_{0}\right) \cdot u \cdot \frac{\operatorname{Cov}\left(\tilde{Z}_{i}, \tilde{\Delta} u\right)}{\operatorname{Var}(\tilde{\Delta} u)}
$$




\section{Remark}

If the $\tilde{Z}_{i}^{\prime}$ s are uncorrelated the fair loading amounts to the variance principle. The multiple of the variance, which must be loaded, is derived from the company portfolio, capitalization level and return objective:

$$
\left(\rho-\rho_{0}\right) \cdot u \cdot \operatorname{Var}^{-1}(\tilde{\Delta} u) \text {. }
$$

If in addition the amount of equity is optimal

$$
u=\tau^{-1} \frac{\operatorname{Var}(\tilde{\Delta} u)}{\left(\rho-\rho_{0}\right) u}
$$

the loading factor is equal to $(\tau u)^{-1}$.

\subsection{Portfolio Optimization}

The excess profit of the company is

$$
\begin{aligned}
\tilde{\Delta} u-\rho_{0} u= & \sum_{i=1}^{m} \alpha_{i} \cdot\left(E\left(\tilde{X}_{i}\right)+\ell_{i}-\tilde{X}_{i}\right) \\
& +\sum_{j=1}^{m^{\prime}} \beta_{j} \cdot\left(\left(\ell_{j}^{\prime}-\tilde{X}_{j}^{\prime}\right)-\left(\tilde{R}_{j}^{\prime}-\rho_{0}\right) \cdot L_{j}\right) \\
& +\sum_{i=1}^{n}\left(\tilde{R}_{j}^{\prime}-\rho_{0}\right) \cdot A_{j}
\end{aligned}
$$

and our objective is to maximize the risk return ratio of the company.

In a first step we have to maximize the risk return ratio of the underwriting and loss reserve subportfolio through reinsurance buying. This leads to more homogeneous and less catastrophe exposed portfolios and hence to higher risk return ratios of the subportfolios. It also leads to distributions which are close to multivariate normal. This process is discussed in section 2 .

We now turn to the second step which consists in the optimization of the global portfolio, i.e. in maximizing the risk return ratio as a function of the $\alpha^{\prime} s, \beta^{\prime} s$ and $A^{\prime} s$.

Let

$$
\begin{aligned}
\underline{x}^{\prime}= & \left(\alpha_{1}, \ldots, \alpha_{m}, \beta_{1}, \ldots, \beta_{m}, A_{1}, \ldots, A_{n}\right) \\
\underline{\mu}^{\prime}= & \left(\ell_{1}, \ldots, \ell_{n}, \ell_{1}^{\prime}-\left(R_{1}^{\prime}-\rho_{0}\right) \cdot L_{1}, \ldots, \ell_{m^{\prime}}^{\prime}-\left(R_{m^{\prime}}^{\prime}-\rho_{0}\right) \cdot L_{m^{\prime}},\right. \\
& \left.R_{1}-\rho_{0}, \ldots, R_{n}-\rho_{0}\right) \\
\Sigma= & \operatorname{Cov}\left(-\tilde{X}_{1}, \ldots,-\tilde{X}_{m},-\tilde{X}_{1}^{\prime}-\tilde{R}_{1}^{\prime} L_{1}, \ldots,-\tilde{X}_{m^{\prime}}-\tilde{R}_{m^{\prime}}^{\prime} L_{m^{\prime}}, \tilde{R}_{1}, \ldots, \tilde{R}_{n}\right)
\end{aligned}
$$


The optimization problem now reads

$$
r=\frac{\underline{x}^{\prime} \cdot \mu}{\left(\underline{x}^{\prime} \cdot \Sigma \cdot \underline{x}\right)^{\frac{1}{2}}}=\max _{\underline{x}} !
$$

with the conditions

$$
\begin{aligned}
x_{i} & =\alpha_{i} \in[0,1] & & i=1, \ldots, m \\
x_{i} & =\beta_{i} \in[0,1] & i & =m+1, \ldots, m+m^{\prime}
\end{aligned}
$$

and if the company is not able to issue securities

$$
x_{i}=A_{i} \geq 0 \quad i=m+m^{\prime}+1, \ldots, m+m^{\prime}+n
$$

This is a standard mathematical programming problem. The solution of which can be derived through standard algorithms.

\section{Remarks}

1. We restrict the reinsurance agreements to genuine quota shares. The company is not allowed to take a short position in any insurance subportfolio - which would be unrealistic - or to increase its share of any insurance subportfolio beyond $100 \%$ - which would attract important acquisition costs.

2. In order for any portfolio to be feasible the amount of liabilities must exceed the amount of assets

$$
u+\sum_{i=1}^{m} \alpha_{i} L_{i} \geq \sum_{i=1}^{n} A_{j}
$$

If this is a true inequality, the assets corresponding to the excess liabilities can be invested in the risk free asset. This amounts to a restriction in the choice of the amount of capital

$$
u \geq \sum_{i=1}^{n} A_{j}-\sum_{i=1}^{m} \alpha_{i} L_{i}
$$

We refer to the right hand side of the inequality as to the amount of net invested assets.

3. Within the framework of our model we can simultaneously optimize the reinsurance policy and the investment policy of the company. The model allows for a symmetrical treatment of the insurance risks and of the asset risks. 


\section{Theorem}

We assume that $\Sigma$ is a regular matrix

1. The unrestricted optimum, i.e. the vector $\underline{x}$ which maximizes

$$
r=\frac{\underline{\mu}^{\prime} \underline{x}}{\left(\underline{x}^{\prime} \sum \underline{x}\right)^{\frac{1}{2}}}
$$

is given by

$$
\underline{x}=c \cdot \Sigma^{-1} \underline{\mu}
$$

(By definition $\underline{x}$ is only defined up to a constant factor $c$.)

The unrestricted optimal risk return ratio is equal to

$$
r_{\max }=\left(\underline{\mu}^{\prime} \Sigma^{-1} \underline{\mu}\right)^{\frac{1}{2}}
$$

2. $\underline{x}$ is the unrestricted optimum if and only if all the actual loadings are equal to the fair loadings.

\section{Proof}

1. We have to maximize

$$
r=\frac{\underline{\mu}^{\prime} \underline{x}}{\left(\underline{x}^{\prime} \Sigma x\right)^{\frac{1}{2}}}
$$

equating to derivatives with respect to $x_{i}$ to zero, we obtain

$$
\frac{d r}{d x_{i}}=\frac{\mu_{i}\left(\underline{x}^{\prime} \Sigma \underline{x}\right)^{\frac{1}{2}}-\underline{\mu}^{\prime} \underline{x} \cdot \frac{1}{2}\left(\underline{x}^{\prime} \Sigma \underline{x}\right)^{-\frac{1}{2} \cdot 2\left(\sum_{j} \sigma_{i j} x_{j}\right)}}{\underline{x}^{\prime} \Sigma \underline{x}}=0
$$

$i=1, \ldots, m+n$ where $\left(\sigma_{i j}\right)=\Sigma$.

After rearranging terms

$$
\begin{gathered}
\mu_{i}\left(\underline{x}^{\prime} \Sigma \underline{x}\right)=\left(\underline{\mu}^{\prime} \underline{x}\right) \sum_{j} \sigma_{i j} x_{j}, \quad \text { all } i \\
\underline{\mu}=k \cdot \Sigma \cdot \underline{x}
\end{gathered}
$$

and since $\Sigma$ is regular

$$
\underline{x}=c \cdot \Sigma^{-1} \underline{\mu}
$$

Plugging in the above definition of $\underline{x}$ we obtain

$$
r_{\max }=\frac{c \cdot \underline{\mu}^{\prime} \Sigma^{-1} \underline{\mu}}{\left(c \underline{\mu}^{\prime} \Sigma^{-1} \cdot \Sigma \cdot c \Sigma^{-1} \underline{\mu}\right)^{\frac{1}{2}}}=\left(\underline{\mu}^{\prime} \Sigma^{-1} \underline{\mu}\right)^{\frac{1}{2}}
$$


2. All the actual loadings are equal to the fair loadings if and only if the following equations are satisfied

$$
\begin{gathered}
\alpha_{i}\left(\ell_{i}+\ell_{i}^{\prime}\right)=k \cdot \operatorname{Cov}\left(-\alpha_{i}\left(\tilde{X}_{i}+\tilde{X}_{i^{\prime}}\right), \tilde{\Delta} u\right) \quad i=1, \ldots, m \\
\left(A_{1}-L\right)\left(R_{1}-\rho_{0}\right)=k \cdot \operatorname{Cov}\left(\left(A_{1}-L\right) \tilde{R}_{1}, \tilde{\Delta} u\right) \\
A_{j}\left(R_{j}-\rho_{0}\right)=k \cdot \operatorname{Cov}\left(A_{j} \tilde{R}_{j}, \tilde{\Delta} u\right) \quad j=2, \ldots, n
\end{gathered}
$$

Using the above notation, this is equivalent to

$$
x_{i} \mu_{i}=k \cdot \operatorname{Cov}\left(x_{i} \tilde{Z}_{i}, \tilde{\Delta} u\right) \quad i=1, \ldots, m+n
$$

for an appropriate choice of $\tilde{Z}_{i}$.

Hence

$$
\begin{gathered}
\mu_{i}=k \cdot \sum_{j} \sum_{i j} x_{j} \quad i=1, \ldots, m+n \\
\underline{\mu}=k \cdot \Sigma \cdot \underline{x}
\end{gathered}
$$

which proves the 2 nd statement of the theorem

q.e.d.

\section{Remarks}

1. The 2 nd statement of our theorem is a further justification for our capital allocation formula.

2. The theorem is a generalisation of the theorem of section 2.5 .

\section{Example 1}

We now turn to a numerical example. The company has two underwriting risks and two loss reserve risks which correspond to the different customer segments of the company. The risks and returns are as follows

\begin{tabular}{lcccc}
\hline \multicolumn{1}{c}{ Underwriting portfolio } & Risk & $\ell$ & $\boldsymbol{\sigma}$ & $\frac{\ell}{\sigma}$ \\
\hline Private customers & $\tilde{X}_{1}$ & 4.5 & 15 & $30 \%$ \\
Industrial customers & $\tilde{X}_{2}$ & 14.4 & 30 & $48 \%$ \\
\hline
\end{tabular}

Note that we do not give the premium income since it is irrelevant. Let $\operatorname{Corr}\left(\tilde{X}_{i}, \tilde{X}_{j}\right)=\delta_{i j}$ where $\delta_{i j}$ is the Kronecker Symbol

$$
\delta_{i j}= \begin{cases}1 & \text { if } i=j \\ 0 & \text { else }\end{cases}
$$




\begin{tabular}{lccccc}
\hline \hline Loss Reserve Portfolio & Risk & $\boldsymbol{L}$ & $\ell$ & $\boldsymbol{\sigma}$ & $\frac{\ell}{\sigma}$ \\
\hline Private customers & $\tilde{X}_{1}$ & 400 & 0.5 & 5 & $10 \%$ \\
Industrial customers & $\tilde{X}_{2}$ & $\frac{600}{1} 000$ & 1.6 & 10 & $16 \%$ \\
\hline
\end{tabular}

with $\operatorname{Corr}\left(\tilde{X}_{i}^{\prime}, \tilde{X}_{j}^{\prime}\right)=\delta_{i j} \quad$ and $\operatorname{Corr}\left(\tilde{X}_{i}, \tilde{X}_{j}^{\prime}\right)=\delta_{i j} \cdot 0.40$.

Note that both in the case of the private customer and of the industrial customer portfolio the ratio between loading and variance is the same for the underwriting and for the loss reserve risk.

There are four different asset categories with risks and returns as defined below

\begin{tabular}{lcccc}
\hline \hline \multicolumn{1}{c}{ Asset Category } & Risk & $\boldsymbol{R}_{\boldsymbol{i}}-\rho_{\mathbf{0}}$ & $\boldsymbol{\sigma}$ & $\frac{\boldsymbol{R}_{\boldsymbol{i}}-\boldsymbol{\rho}_{\mathbf{0}}}{\boldsymbol{\sigma}}$ \\
\hline $\begin{array}{l}\text { Bond portfolio with medium } \\
\text { term duration }\left(\tilde{R}_{1}=\tilde{R}_{L}\right)\end{array}$ & $\tilde{R}_{1}$ & $1 \%$ & $4 \%$ & $25 \%$ \\
$\begin{array}{l}\text { Bond portfolio with long term } \\
\text { duration }\end{array}$ & $\tilde{R}_{2}$ & $2 \%$ & $6 \%$ & $33 \%$ \\
Equity portfolio & $\tilde{R}_{3}$ & $10 \%$ & $20 \%$ & $50 \%$ \\
Real Estate portfolio & $\tilde{R}_{4}$ & $8 \%$ & $20 \%$ & $40 \%$ \\
\hline
\end{tabular}

The correlation matrix of the different asset categories is as follows

$$
\operatorname{Corr}\left(\tilde{R}_{i}, \tilde{R}_{j}\right)=\left[\begin{array}{cccc}
1 & 0.9 & 0.4 & 0.4 \\
& 1 & 0.4 & 0.4 \\
& & 1 & 0.4 \\
& & & 1
\end{array}\right]
$$

It is assumed that insurance risks and asset risks are uncorrelated

$$
\operatorname{Corr}\left(\tilde{X}_{i}, \tilde{R}_{j}\right)=0 \quad \text { for all } i, j .
$$

Without any loss of generality we assume

$$
\tilde{R}_{i}=\tilde{R}_{i}^{\prime} \quad i=1,2 .
$$

This amounts to choosing bond portfolios with maturities matching the expected maturities of the respective liability portfolios.

We have

$$
\underline{\mu}^{\prime}=(4.5,14.4,-3.5,-10.4,0.01,0.02,0.10,0.08)
$$


and

$$
\begin{aligned}
& \Sigma=\left[\begin{array}{ccc}
\operatorname{Cov}\left(\tilde{X}_{i}, \tilde{X}_{j}\right) & \operatorname{Cov}\left(\tilde{X}_{i}^{\prime}, \tilde{X}_{j}^{\prime}\right) & 0 \\
\operatorname{Cov}\left(\tilde{X}_{i}^{\prime}, \tilde{X}_{j}\right) & \operatorname{Cov}\left(\tilde{X}_{i}^{\prime}, \tilde{X}_{j}^{\prime}\right)+L_{i} L_{j} \cdot \operatorname{Cov}\left(\tilde{R}_{i}^{\prime}, \tilde{R}_{j}\right) & -L_{i} \cdot \operatorname{Cov}\left(\tilde{R}_{i}^{\prime}, \tilde{R}_{j}\right) \\
0 & -L_{j} \cdot \operatorname{Cov}\left(\tilde{R}_{i}, \tilde{R}_{j}^{\prime}\right) & \operatorname{Cov}\left(\tilde{R}_{i}, \tilde{R}_{j}\right)
\end{array}\right] \\
& \Sigma=\left[\begin{array}{rrllllll}
225 & 0 & 30 & 0 & 0 & 0 & 0 & 0 \\
0 & 900 & 0 & 120 & 0 & 0 & 0 & 0 \\
30 & 0 & 281 & 518.4 & -0.64 & -0.864 & -1.28 & -1.28 \\
0 & 120 & 518.4 & 1396 & -1.296 & -2.16 & -2.88 & -2.88 \\
0 & 0 & -0.64 & -1.296 & 0.0016 & 0.00216 & 0.0032 & 0.0032 \\
0 & 0 & -0.864 & -2.16 & 0.00216 & 0.0036 & 0.0048 & 0.0048 \\
0 & 0 & -1.28 & -2.88 & 0.0032 & 0.0048 & 0.04 & 0.016 \\
0 & 0 & -1.2 & -2.88 & 0.0032 & 0.0048 & 0.016 & 0.04
\end{array}\right]
\end{aligned}
$$

The unconstrained solution is

$$
\underline{x}=c \cdot \Sigma^{-1} \cdot \underline{\mu}=(1,0.8,-0.23,-0.18,-568.1,245.1,94.8,54.4)^{\prime}
$$

which is not admissible because it entails taking a short position in the two loss reserve risks and issuing the short term bond portfolio for an amount of 568.1 monetary units. The constrained optimization problem is

$$
c \cdot \underline{x}^{\prime} \cdot \underline{\mu}-\underline{x}^{\prime} \cdot \Sigma^{-1} \cdot \underline{x}=\max _{\underline{x}}
$$

with

$$
x_{3}=x_{4}=x_{5}=0
$$

The associated objective function is

$$
Z=c \cdot \underline{x}^{\prime} \cdot \underline{\mu}-\underline{x}^{\prime} \cdot \Sigma \cdot \underline{x}+\lambda_{3} x_{3}+\lambda_{4} x_{4}+\lambda_{5} x_{5}=\max !
$$

where $\lambda_{3}, \lambda_{4}$ and $\lambda_{5}$ are the Lagrange multipliers associated with the above constraints. To solve the constrained optimization problem we must find $x_{1}, \ldots, x_{N}\left(N=m+m^{\prime}+n\right)$ such that

$$
\frac{\partial Z}{\partial x_{i}}=0 \quad i=1, \ldots, N \quad \text { and } \quad \frac{\partial Z}{\partial \lambda_{3}}=\frac{\partial Z}{\partial \lambda_{4}}=\frac{\partial Z}{\partial \lambda_{5}}=0 .
$$

This leads to the following set of equations

$$
\begin{gathered}
-c \cdot \mu_{i}+2 \sum_{j=1}^{N} \sigma_{i j} \cdot x_{j}-\sum_{j=3}^{5} \lambda_{j} \delta_{i, j}=0 \\
x_{3}=x_{4}=x_{5}=0
\end{gathered}
$$


in matrix notation

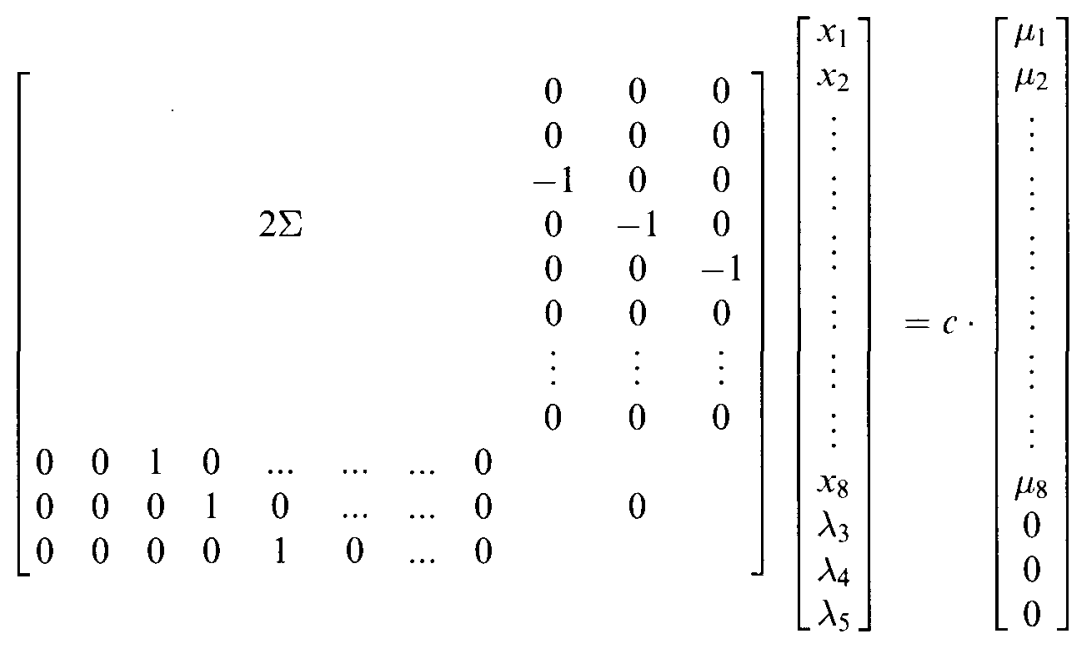

or

$$
\Sigma^{*} \underline{x}^{*}=\underline{\mu}^{*}
$$

and the solution is

$$
\underline{x} *=c\left(\Sigma^{*}\right)^{-1} \underline{\mu}^{*}
$$

\begin{tabular}{|c|c|c|c|}
\hline Underwriting Risk & Coefficient $\alpha, \beta$ or $A$ & Expected Profit & $\begin{array}{l}\text { Contribution to } \\
\text { overall variance }\end{array}$ \\
\hline$-\tilde{X}_{1}+\ell_{1}+E\left(\tilde{X}_{1}\right)$ & 1 & 4.5 & 225.00 \\
\hline$-\tilde{X}_{2}+\ell_{2}+E\left(\tilde{X}_{2}\right)$ & 0.80 & 11.52 & 576.00 \\
\hline \multicolumn{4}{|l|}{ Loss Reserve Risk } \\
\hline$-\tilde{X}_{1}^{\prime}+\ell_{1}^{\prime}-\left(\tilde{R}_{1}^{\prime}-\rho_{0}\right) \cdot L_{1}$ & 0 & 0 & 0 \\
\hline$-\tilde{X}_{2}^{\prime}+\ell_{2}^{\prime}-\left(\tilde{R}_{2}^{\prime}-\rho_{0}\right) \cdot L_{2}$ & 0 & 0 & 0 \\
\hline \multicolumn{4}{|l|}{ Asset Risks } \\
\hline$\overline{\tilde{R}_{1}-\rho_{0}}$ & 0 & 0 & 0 \\
\hline$\tilde{R}_{2}-\rho_{0}$ & 82.30 & 1.65 & 82.30 \\
\hline$\tilde{R}_{3}-\rho_{0}$ & 94.14 & 9.41 & 470.68 \\
\hline \multirow[t]{2}{*}{$\tilde{R}_{4}-\rho_{0}$} & 52.47 & 4.20 & 209.88 \\
\hline & & $\overline{31.28}$ & $\overline{1563.86}$ \\
\hline
\end{tabular}

The optimal constrained portfolio of the company is thus 
The optimal amount of capital is

$$
u=\tau^{-1} \frac{V}{R}
$$

with $R$ and $V$ are the expected profit and the contribution to overall variance respectively (see section 2.2.). Assuming $\tau$ we obtain $u=199.98$.

The salient features of the optimal portfolio are the following

- The company cedes a $20 \%$ quota share of its industrial business.

- The company fully reinsures the loss reserve risk. As a consequence its balance sheet is not leveraged at all. The liability side of the balance sheet consists of equity only, there is no debt.

- The total amount of net invested assets is 228.91 which compares with an optimal amount of equity of 199.98. The optimal policy is only feasible if the company can raise an amount of debt of 28.93 monetary units at the risk free rate.

- The company invests a substantial part of its nets invested assets in shares and real estate $(64 \%)$. The contribution to the expected profit and to the overall volatility from asset risks is substantial (49\%).

- The optimal risk return ratio is $r=0.791$.

- For the unconstrained risks (i.e. all the risks except $x_{3}, x_{4}$ and $x_{5}$ ) we have

$$
\frac{\text { expected profit }}{\text { contribution to overall variance }}=\text { constant }=0.020 \text {. }
$$

For the constrained risks the above quantity is irrelevant.

\section{Example 2}

Based on the result of the section on loss reserves, the model assumes that the loadings $\ell_{i}$ and $\ell_{i}^{\prime}$ are proportional to the variance of the corresponding risks $\sigma^{2}\left(\tilde{X}_{i}\right)$ and $\sigma^{2}\left(\tilde{X}_{i}^{\prime}\right)$. In practice however a loss portfolio transfer $\left(\beta_{i}=0\right)$ would probably command a much higher loading. Since there is no liquid reinsurance market for loss portfolio transfers we make the following

\section{Assumption 7}

$m^{\prime}=m, \beta_{i}=\alpha_{i} \quad i=1, \ldots, m$.

In addition we simplify the notation

$\tilde{X}_{i}+\tilde{X}_{i}^{\prime}$ is replaced by $\tilde{X}_{i}$, and

$\ell_{i}+\ell_{i}^{\prime}$ is replaced by $\ell_{i}$.

The model now becomes

$$
\tilde{\Delta} u-\rho_{0} u=\sum_{i=1}^{m} \alpha_{i}\left(E\left(\tilde{X}_{i}\right)+\ell_{i}-\tilde{X}_{i}-\left(\tilde{R}_{i}^{\prime}-\rho_{0}\right) \cdot L_{i}\right)+\sum_{j=1}^{m}\left(\tilde{R}_{j}-\rho_{0}\right) \cdot A_{j}
$$


We now reanalyze the preceding example. We have

\begin{tabular}{lccccc}
\hline \multicolumn{1}{c}{ Insurance Portfolio } & Risk & $\boldsymbol{L}$ & $\boldsymbol{\ell}$ & $\boldsymbol{\sigma}$ & $\frac{\ell}{\sigma}$ \\
\hline Private customers & $\tilde{X}_{1}^{\prime}$ & 400 & 5 & 17.61 & $28 \%$ \\
Industrial customers & $\tilde{X}_{2}^{\prime}$ & 600 & 16 & 35.12 & $45 \%$ \\
\hline \hline
\end{tabular}

The other model parameters remain unchanged and we have

$$
\begin{gathered}
\mu^{\prime}=(1,4,0.01,0.02,0.10,0.08) \\
\Sigma=\left[\begin{array}{ccccl}
\operatorname{Cov}\left(\tilde{X}_{i}, \tilde{X}_{j}\right)+L_{i} L_{j} \operatorname{Cov}\left(\tilde{R}_{i}^{\prime}, \tilde{R}_{j}^{\prime}\right) \\
-L_{i} \operatorname{Cov}\left(\tilde{R}_{j}, \tilde{R}_{i}^{\prime}\right) & \multicolumn{3}{r}{\operatorname{Cov}\left(\tilde{R}_{i}^{\prime}, \tilde{R}_{j}\right)} \\
\operatorname{Cov}\left(\tilde{R}_{i}, \tilde{R}_{j}\right)
\end{array}\right] \\
\Sigma=\left[\begin{array}{llllll}
566 & 518.4 & -0.64 & -0.864 & -1.28 & -1.28 \\
518.4 & 2536 & -1.296 & -2.16 & -2.88 & -2.88 \\
-0.64 & -1.296 & 0.0016 & 0.00216 & 0.0032 & 0.0032 \\
-0.864 & -2.16 & 0.00216 & 0.0036 & 0.0048 & 0.0048 \\
-1.28 & -2.88 & 0.0032 & 0.0048 & 0.04 & 0.016 \\
-1.28 & -2.88 & 0.0032 & 0.0048 & 0.016 & 0.04
\end{array}\right]
\end{gathered}
$$

The unconstrained solution is

$$
\underline{x}=c \cdot \Sigma^{-1} \underline{\mu}=(1,0.8,-208.7,935.3,121.2,69.6)
$$

which entails a short position of 208.7 monetary units in the medium term bond (in addition to the 400 monetary units of loss reserves with the same return $\tilde{R}_{1}^{\prime}=\tilde{R}_{1}$ ). Within the framework of this model this is not admissible. We therefore introduce the side condition

$$
x_{3}=0
$$

which leads to the following objective function

$$
Z=c \cdot \underline{x}^{\prime} \cdot \underline{\mu}-\underline{x^{\prime}} \Sigma \underline{x}+\lambda_{3} x_{3}=\max !
$$


Deriving with respect to $x_{i}$ and $\lambda_{3}$, we obtain the following matrix equation

$$
\left[\begin{array}{ccccccc} 
& & & & & & \\
& & & & & 0 \\
& & & & \\
& & & 0 \\
& & & & & & 0 \\
& & & & & & 0 \\
0 & 0 & 1 & 0 & 0 & 0 & 0
\end{array}\right]\left[\begin{array}{c}
x_{1} \\
\vdots \\
\vdots \\
\vdots \\
\vdots \\
\vdots \\
x_{6} \\
\lambda_{3}
\end{array}\right]=c \cdot\left[\begin{array}{c}
\mu_{1} \\
\vdots \\
\vdots \\
\vdots \\
\vdots \\
\vdots \\
\mu_{6} \\
0
\end{array}\right]
$$

which is easily solved yielding the following optimal constrained portfolio

\begin{tabular}{|c|c|c|c|c|}
\hline Insurance Risk & Coefficients $\alpha_{i}$ & $L$ & $\begin{array}{c}\text { Expected } \\
\text { profit }\end{array}$ & $\begin{array}{l}\text { Contribution to } \\
\text { overall variance }\end{array}$ \\
\hline$-\tilde{X}_{1}^{\prime}+\ell_{1}^{\prime}-\left(\tilde{R}_{1}^{\prime}-\rho_{0}\right) \cdot L_{1}$ & 1 & 400 & 1 & 57.95 \\
\hline \multirow[t]{2}{*}{$-\tilde{X}_{2}^{\prime}+\ell_{2}^{\prime}-\left(\tilde{R}_{2}^{\prime}-\rho_{0}\right) \cdot L_{2}$} & 0.75 & $\underline{450}$ & 2.99 & 173.35 \\
\hline & & 850 & & \\
\hline Asset Risks & $\begin{array}{c}\text { Invested } \\
\text { Amounts } A_{j}\end{array}$ & & $\begin{array}{c}\text { Expected } \\
\text { profit }\end{array}$ & $\begin{array}{l}\text { Contribution to } \\
\text { overall variance }\end{array}$ \\
\hline $\bar{R}_{1}-\rho_{0}$ & 0 & & 0 & 0 \\
\hline$\tilde{R}_{2}-\rho_{0}$ & 776.2 & & 15.52 & 899.64 \\
\hline$\tilde{R}_{3}-\rho_{0}$ & 112.1 & & 11.21 & 649.50 \\
\hline \multirow[t]{2}{*}{$\tilde{R}_{4}-\rho_{0}$} & 63.8 & & 5.10 & 295.70 \\
\hline & $\overline{952.1}$ & & $\overline{35.82}$ & $\overline{2076.14}$ \\
\hline
\end{tabular}

Assuming $\tau=0.25$, the optimal amount of equity is

$$
u=\tau^{-1} \frac{V}{R}=231.83
$$

The salient features of the optimal portfolio are the following

- The company cedes a $25 \%$ quota share of its industrial business.

- The company keeps most of its loss reserves (850 monetary units out of a gross amount of 1000) thus leveraging its balance sheet.

- The total amount of net invested assets is 102.1 which compares with an optimal amount of equity of 231.83 . The optimal policy is thus feasible without borrowing. 
- The contribution to the expected profit and to the overall volatility from asset risks is much higher than the corresponding quantities from insurance risks ( $89 \%$ vs $11 \%$ ). This is in particular due to the fact that the short position in yield curve risk acts as a hedge.

- The optimal risk return ratio $r=0.786$.

- For unconstrained risks, we have

$$
\frac{\text { expected profit }}{\text { contribution to overall variance }}=\text { constant }=0.017
$$

\section{Example 3}

So far we have assumed that the company may not issue securities, or in other words that $A_{i} \geq 0 i=1, \ldots, n$.

We now make the following

\section{Assumption 8}

The company may issue securities, i.e. $A_{i} i=1, \ldots, n$ are unconstrained.

Without loss of generality we also assume $n \geq m$ and

$$
\tilde{R}_{i}^{\prime} \equiv \tilde{R}_{i} \quad i=1, \ldots, m
$$

and we introduce the following notational simplification

$$
B_{j}=A_{j}-\alpha_{j} L_{j} \quad j=1, \ldots, m
$$

The model can now be rewritten as

$$
\tilde{\Delta} u-\rho_{0} \cdot u=\sum_{i=1}^{m} \alpha_{i}\left(E\left(\tilde{X}_{i}\right)+\ell_{i}-\tilde{X}_{i}\right)+\sum_{j=1}^{n}\left(\tilde{R}_{j}-\rho_{0}\right) \cdot B_{j} .
$$

We have

$$
\begin{gathered}
\underline{\mu}^{\prime}=(5,16,0.01,0.02,0.10,0.08) \\
\Sigma=\left[\begin{array}{rllll}
\operatorname{Cov}\left(\tilde{X}_{i}, \tilde{X}_{j}\right) & 0 \\
0 & \operatorname{Cov}\left(\tilde{R}_{i}, \tilde{R}_{j}\right)
\end{array}\right] \\
\Sigma=\left[\begin{array}{rlllll}
310 & 0 & 0 & 0 & 0 & 0 \\
0 & 1240 & 0 & 0 & 0 & 0 \\
0 & 0 & 0.0016 & 0.00216 & 0.0032 & 0.0032 \\
0 & 0 & 0.00216 & 0.0036 & 0.0048 & 0.0048 \\
0 & 0 & 0.0032 & 0.0048 & 0.04 & 0.016 \\
0 & 0 & 0.0032 & 0.0048 & 0.016 & 0.04
\end{array}\right]
\end{gathered}
$$


The unconstrained solution

$$
\underline{x}=c \cdot \Sigma^{-1} \underline{\mu}=(1,0.8,-608.7,455.3,121.2,69.6)
$$

is now admissible and the optimal portfolio is

\begin{tabular}{lccc}
\hline Insurance Risks & Coefficients $\boldsymbol{\alpha}_{\boldsymbol{i}}$ & Expected profit & $\begin{array}{c}\text { Contribution to } \\
\text { overall variance }\end{array}$ \\
\hline$E\left(\tilde{X}_{1}\right)+\ell_{1}-\tilde{X}_{1}$ & 1 & 5 & 310 \\
$E\left(\tilde{X}_{2}\right)+\ell_{2}-\tilde{X}_{2}$ & 0.8 & 12.8 & 793.6 \\
\hline Financial Risks & Net invested Amounts $\boldsymbol{B}_{\boldsymbol{j}}$ & & \\
\hline$\tilde{R}_{1}-\rho_{0}$ & -608.7 & -6.09 & -377.4 \\
$\tilde{R}_{2}-\rho_{0}$ & 455.3 & 9.11 & 564.5 \\
$\tilde{R}_{3}-\rho_{0}$ & 121.2 & 12.12 & 751.7 \\
$\tilde{R}_{4}-\rho_{0}$ & 69.6 & 5.57 & 345.1 \\
& 37.4 & 38.51 & 2387.5 \\
\hline \hline
\end{tabular}

Assuming $\tau=0.25$, the optimal amount of equity is

$$
u=\tau^{-1} \frac{V}{R}=247.99
$$

The salient features of the optimal portfolio are

- The company cedes a $20 \%$ quota share of its industrial business.

- The gross invested amounts in asset category 1 and 2 are respectively -208.7 and 935.3 which are identical with the corresponding amounts pertaining to the (inadmissible) unconstrained solution of the preceding example.

- The amount of net invested assets is 37.4 which compares with an optimal amount of equity of 231.83 .

- The contribution to the expected profit and to the overall volatility from financial risks (including short position in yield curve risk) is higher than the corresponding quantities from insurance risks ( $54 \%$ vs $46 \%$ ).

- The optimal risk return ratio is $r=0.788$.

- The ratio of expected profit to contribution to overall variance is the same for all risks (0.016).

\section{Discussion of Assumption 8}

A comparison between the last two examples shows that dropping the constraint $A_{j} \geq 0$ (for all $j$ ) leads to a higher risk return ratio and to a lower amount of net invested assets. In practice insurance companies are allowed to issue preferred shares or - through a holding company - obtain bank loans or issue corporate bonds. The amount of debt they are able to raise is usually limited and commands a spread over the risk free rate. 


\section{Generalization of Theorem 4.2}

From the above example it is seen that the constrained optimum is obtained by computing the unconstrained solution

$$
\underline{x}=c \cdot \Sigma^{-1} \cdot \underline{\mu}
$$

and by choosing $c$ in such a way that the retained insurance profit is maximized (i.e. $\max _{i=1, \ldots, m+m^{\prime}} x_{j}=1$ ).

Let $i_{1}, \ldots, i_{k}$ be those indices for which $x_{i}<0$. The constrained optimum is obtained by maximizing the following objective function

$$
Z=\lambda \underline{x^{\prime}} \cdot \underline{\mu}-\underline{x}^{\prime} \Sigma \underline{x}-\lambda_{i_{1}} x_{i_{1}}-\ldots-\lambda_{i_{k}} x_{i_{k}}
$$

where $\lambda_{i_{1}}, \ldots, \lambda_{i_{k}}$ are the Lagrange multipliers associated with the constraints

$$
x_{i_{j}}=0 \quad(j=1, \ldots, k) .
$$

This leads to the following set of equations

$$
\begin{gathered}
\frac{\partial Z}{\partial x_{i}}=-\lambda \mu_{i}+2 \sum_{j} \sigma_{i j} x_{j}-\sum_{j=1}^{k} \delta_{i, i_{j}} \lambda_{j}=0 \\
x_{i_{1}}=\ldots=x_{i_{k}}=0
\end{gathered}
$$

In particular for unconstrained variables $x_{i}$, we have

$$
\frac{2}{\lambda} \cdot \sum_{j} \sigma_{i j} x_{j}=\mu_{i}
$$

which translates into

$$
\begin{gathered}
\ell_{i}=k \cdot \operatorname{Cov}\left(-\tilde{X}_{i}, \tilde{\Delta} u\right) \\
\ell_{i}^{\prime}-\left(R_{i}^{\prime}-\rho_{0}\right) L_{i}=k \cdot \operatorname{Cov}\left(-\tilde{X}_{i}^{\prime}-\tilde{R}_{i}^{\prime} \cdot L_{i}, \tilde{\Delta} u\right) \\
R_{i}-\rho_{0}=k \cdot \operatorname{Cov}\left(\tilde{R}_{i}, \tilde{\Delta} u\right)
\end{gathered}
$$

i.e. the loading pertaining to unconstrained variables is equal to the fair loading. This is a further justification for our capital allocation and pricing formula. 


\subsection{Insurance Risk and Financial Risk}

We consider the expression for the excess profit of the company which we have derived at the beginning of section 4 .

$$
\begin{aligned}
\tilde{\Delta} u-\rho_{0} u & =E(\tilde{S})+\ell+\ell_{1}-\left(\tilde{S}+\tilde{\Delta} L_{1}\right)-\left(\tilde{R}_{L}-\rho_{0}\right) L+\sum_{j=1}^{n}\left(\tilde{R}_{j}-\rho_{0}\right) A_{j} \\
& =\tilde{Z}+\left(\tilde{R}-\rho_{0}\right) A
\end{aligned}
$$

where

$$
\begin{gathered}
\tilde{Z}=E(\tilde{S})+\ell+\ell_{1}-\left(\tilde{S}+\tilde{\Delta} L_{1}\right) \\
\tilde{R}=\frac{\sum_{j} \tilde{R}_{j} \cdot A_{j}-R_{L} \cdot L}{A} \text { with } A=\sum_{j=1}^{n} A_{j}-L
\end{gathered}
$$

$\tilde{Z}$ is the insurance risk, i.e. the sum of the underwriting risk and of the loss reserve development risk. $\tilde{R}$ is the rate of return of the financial risk and $A$ is the amount of net invested assets. We introduce the following notation

$$
\begin{gathered}
\ell_{z}=E(\tilde{Z})=\ell+\ell_{1}, \quad \sigma_{z}^{2}=\operatorname{Var}(\tilde{Z}) \\
\delta_{R}=E(\tilde{R})-\rho_{0}, \quad \sigma_{R}^{2}=\operatorname{Var}(\tilde{R}), \quad \mathcal{K}=\operatorname{Corr}(\tilde{Z}, \tilde{R})
\end{gathered}
$$

The following theorem expresses the overall risk return ratio as a function of the insurance risk return ratio and of the financial risk return ratio.

\section{Theorem}

Let $\mathcal{K} \neq \pm 1$. The overall risk return ratio

$$
r(A)=\frac{E(\tilde{\Delta} u)-\rho_{0} u}{\sigma(\tilde{\Delta} u)}=\frac{\ell_{z}+\delta_{R} A}{\sqrt{\sigma_{z}^{2}+\left(\sigma_{R} A\right)^{2}+2 \mathcal{K} \sigma_{z}\left(\sigma_{R} A\right)}}
$$

is maximized for the following amount of net invested assets

$$
A=\frac{\ell_{z}}{\delta_{R}} \frac{\left(\frac{\delta_{R}}{\sigma_{R}}\right)^{2}-\mathcal{K} \frac{\ell_{z}}{\sigma_{z}} \frac{\delta_{R}}{\sigma_{R}}}{\left(\frac{\ell_{z}}{\sigma_{z}}\right)^{2}-\mathcal{K} \frac{\ell_{z}}{\sigma_{z}} \frac{\delta_{R}}{\sigma_{R}}}
$$

and the corresponding risk return ratio is

$$
r=r(A)=\left(\frac{\left(\frac{\ell_{z}}{\sigma_{z}}\right)^{2}+\left(\frac{\delta_{R}}{\sigma_{R}}\right)^{2}-2 \mathcal{K} \frac{\ell_{z}}{\sigma_{z}} \frac{\delta_{R}}{\sigma_{R}}}{1-\mathcal{K}^{2}}\right)^{\frac{1}{2}}
$$




\section{Proof}

We have

$$
E(\tilde{\Delta} u)-\rho_{0} u=\ell_{z}+\delta_{R} \cdot A, \quad \sigma^{2}(\tilde{\Delta} u)=\sigma_{z}^{2}+\sigma_{R}^{2} A^{2}+2 \mathcal{K} \sigma_{z} \sigma_{R} \cdot A
$$

it follows that

$$
r(A)=\frac{\ell_{z}+\delta_{R} A}{\sqrt{\sigma_{z}^{2}+\left(\sigma_{R} A\right)^{2}+2 \mathcal{K} \sigma_{z} \sigma_{R} A}}=\frac{\delta(A)}{\sqrt{V(A)}}
$$

where $\delta(A)$ is $E(\tilde{\Delta} u)-\rho_{0} \cdot u$ and $V(A)$ is $\sigma^{2}(\tilde{\Delta} u)$ considered as function of $A$.

Putting the derivative of $r$ with respect to $A$ equal to zero, we obtain

$$
\begin{gathered}
r^{\prime}(A)=\frac{\delta^{\prime}(A) \cdot V(A)^{\frac{1}{2}}-\delta(A) \frac{1}{2} V(A)^{-\frac{1}{2}} V^{\prime}(A)}{V(A)}=0 \\
\delta_{R}\left(\sigma_{z}^{2}+\sigma_{R}^{2} A^{2}+2 \mathcal{K} \sigma_{z} \sigma_{R} A\right)=\left(\ell_{z}+\delta_{R} A\right)\left(\sigma_{R}^{2} A+\mathcal{K} \sigma_{z} \sigma_{R}\right) \\
A=\frac{\delta_{R} \sigma_{z}^{2}-\mathcal{K} \ell_{z} \sigma_{z} \sigma_{R}}{\ell_{z} \sigma_{R}^{2}-\mathcal{K} \delta_{R} \sigma_{z} \sigma_{R}}=\frac{\ell_{z}}{\delta_{R}} \frac{\left(\frac{\delta_{R}}{\sigma_{R}}\right)^{2}-\mathcal{K} \frac{\ell_{z}}{\sigma_{z} \sigma_{R}}}{\left(\frac{\ell_{z}}{\sigma_{z}}\right)^{2}-\mathcal{K} \frac{\ell_{z}}{\sigma_{z}} \frac{\delta_{R}}{\sigma_{R}}}
\end{gathered}
$$

which proves the first statement of the theorem. In order to evaluate $r(A)$, we introduce the following notation

$$
r_{1}=\frac{\ell_{z}}{\sigma_{z}} \quad r_{2}=\frac{\delta_{R}}{\sigma_{R}}
$$

and we restate the expression for $A$

$$
A=\frac{\sigma_{z}}{\sigma_{R}} \frac{r_{2}-\mathcal{K} r_{1}}{r_{1}-\mathcal{K} r_{2}}
$$

Thus obtaining

$$
\begin{gathered}
V(A)=\sigma_{z}^{2}+\sigma_{z}^{2}\left(\frac{r_{2}-\mathcal{K} r_{1}}{r_{1}-\mathcal{K} r_{2}}\right)^{2}+2 \mathcal{K} \sigma_{z}^{2} \frac{r_{2}-\mathcal{K} r_{1}}{r_{1}-\mathcal{K} r_{2}} \\
V(A)=\frac{\sigma_{z}^{2}}{\left(r_{1}-\mathcal{K} r_{2}\right)^{2}}\left(\left(r_{1}-\mathcal{K} r_{2}\right)^{2}+\left(r_{2}-\mathcal{K} r_{1}\right)^{2}+2 \mathcal{K}\left(r_{2}-\mathcal{K} r_{1}\right)\left(r_{1}-\mathcal{K} r_{2}\right)\right) \\
V(A)=\frac{\sigma_{z}^{2}}{\left(r_{1}-\mathcal{K} r_{2}\right)^{2}}\left(1-\mathcal{K}^{2}\right) \cdot\left(r_{1}^{2}+r_{2}^{2}-2 \mathcal{K} r_{1} r_{2}\right) \\
r(A)=\frac{r_{1}-\mathcal{K} r_{2}}{\sigma_{z}} \frac{\ell_{z}+\delta_{R} \frac{\sigma_{z}}{\sigma_{R}} r_{r_{1}-\mathcal{K} r_{1}}}{\sqrt{\left(1-\mathcal{K}^{2}\right)\left(r_{1}^{2}+r_{2}^{2}-2 \mathcal{K} r_{1} r_{2}\right)}}=\sqrt{\frac{r_{1}^{2}+r_{2}^{2}-2 \mathcal{K} r_{1} r_{2}}{1-\mathcal{K}^{2}}}
\end{gathered}
$$

which proves the theorem.

q.e.d. 


\section{Remarks}

1. From the proof of the theorem it is easily seen that for $\mathcal{K}= \pm 1$ we have $A=\mp \frac{\sigma_{z}}{\sigma_{R}}$ and $V(A)=0$ i.e. the risk if fully eliminated.

2. For $\mathcal{K}=0$ we have

$$
A=\frac{\sigma_{z}}{\sigma_{R}} \frac{\left(\frac{\delta_{R}}{\sigma_{R}}\right)}{\left(\frac{\ell_{z}}{\sigma_{z}}\right)} \quad \text { and } \quad r(A)=\sqrt{\left(\frac{\ell_{z}}{\sigma_{z}}\right)^{2}+\left(\frac{\delta_{R}}{\sigma_{R}}\right)^{2}}
$$

and it is seen that the assumption of asset risk leads to a considerably higher risk return ratio. In practice we have $\mathcal{K} \simeq 0$ and the statement is thus true for all practical situations.

\subsection{Realistic Example}

We now turn to a more realistic example. The insurance portfolio of the company is broken down into four subportfolios corresponding to different lines of business and to different customer segments. The risks and returns of the combined underwriting and loss development risks are as follows

\begin{tabular}{lcccccc}
\hline \multicolumn{1}{c}{ Insurance Subportfolio } & Risks & $\boldsymbol{P}$ & $\boldsymbol{L}$ & $\boldsymbol{\sigma}$ & $\ell$ & $\frac{\ell}{\sigma}$ \\
\hline Motor & $\tilde{X}_{1}$ & 50 & 75 & 2.5 & 0.5 & $20 \%$ \\
Homeowners & $\tilde{X}_{2}$ & 20 & 10 & 3.2 & 0.8 & $25 \%$ \\
Industrial Fire & $\tilde{X}_{3}$ & 10 & 5 & 4 & 1 & $25 \%$ \\
General Third Party Liability & $\tilde{X}_{4}$ & $\underline{\underline{10}}$ & $\underline{20}$ & 4 & $\underline{1.5}$ & $37.5 \%$ \\
& & & & & &
\end{tabular}

$L$ denotes the amount of loss reserves.

The premium volume is given for purely illustrative purposes. It is not used below. The ratio between standard deviation and premium volume as well as the ratio between loss reserves and premium are chosen in a realistic way. It is assumed that the motor and the homeowners portfolio are both exposed to storm and are therefore positively correlated.

$$
\operatorname{Corr}\left(\tilde{X}_{1}, \tilde{X}_{2}\right)=0.20
$$

The other correlations between insurance risks stem from the influence of the economic cycle and are treated below. 
The different asset categories are as in the example of section 4.2.

\begin{tabular}{lcccc}
\hline \hline \multicolumn{1}{c}{ Asset Category } & Risk & $\boldsymbol{R}_{\boldsymbol{i}}-\rho_{\mathbf{0}}$ & $\boldsymbol{\sigma}$ & $\frac{\boldsymbol{R}_{\boldsymbol{i}}-\boldsymbol{\rho}_{\mathbf{0}}}{\boldsymbol{\sigma}}$ \\
\hline $\begin{array}{l}\text { Bond portfolio with medium } \\
\text { term duration }\left(\tilde{R}_{1}=\tilde{R}_{L}\right)\end{array}$ & $\tilde{R}_{1}$ & $1 \%$ & $4 \%$ & $25 \%$ \\
$\begin{array}{l}\text { Bond portfolio with long } \\
\text { term }\end{array}$ & $\tilde{R}_{2}$ & $2 \%$ & $6 \%$ & $33 \%$ \\
$\begin{array}{l}\text { duration } \\
\text { Equity portfolio }\end{array}$ & $\tilde{R}_{3}$ & $10 \%$ & $20 \%$ & $50 \%$ \\
Real Estate portfolio & $\tilde{R}_{4}$ & $8 \%$ & $20 \%$ & $40 \%$ \\
\hline \hline
\end{tabular}

The correlation matrix of the different asset categories is as follows

$$
\operatorname{Corr}\left(\tilde{R}_{i}, \tilde{R}_{j}\right)=\left[\begin{array}{cccc}
1 & 0.9 & 0.4 & 0.4 \\
& 1 & 0.4 & 0.4 \\
& & 1 & 0.4 \\
& & & 1
\end{array}\right]
$$

During a boom phase of the economic cycle interest rates and therefore investment income from bonds are high, but so is the inflation rate which leads to an increased loss amount of the motor and of the general third party liability portfolio. Therefore we assume

$$
\begin{aligned}
& \operatorname{Corr}\left(-\tilde{X}_{1}, \tilde{R}_{1}\right)=\operatorname{Corr}\left(-\tilde{X}_{1}, \tilde{R}_{2}\right)=-0.2 \\
& \operatorname{Corr}\left(-\tilde{X}_{4}, \tilde{R}_{1}\right)=\operatorname{Corr}\left(-\tilde{X}_{4}, \tilde{R}_{2}\right)=-0.2
\end{aligned}
$$

and

$$
\operatorname{Corr}\left(\tilde{X}_{1}, \tilde{X}_{4}\right)=0.2
$$

When the economy goes into recession, equities and real estate depreciate, industrial fire results worsen - due to arson - and motor results improve because people drive less. Thus

$$
\begin{aligned}
& \operatorname{Corr}\left(-\tilde{X}_{1}, \tilde{R}_{3}\right)=\operatorname{Corr}\left(-\tilde{X}_{1}, \tilde{R}_{4}\right)=-0.2 \\
& \operatorname{Corr}\left(-\tilde{X}_{3}, \tilde{R}_{3}\right)=\operatorname{Corr}\left(-\tilde{X}_{3}, \tilde{R}_{3}\right)=0.2
\end{aligned}
$$

and

$$
\operatorname{Corr}\left(\tilde{X}_{1}, \tilde{X}_{3}\right)=-0.2
$$


In summary we have the following correlations

\begin{tabular}{r|cccccccc} 
& $-\tilde{\boldsymbol{X}}_{\mathbf{1}}$ & $-\tilde{\boldsymbol{X}}_{\mathbf{2}}$ & $-\tilde{\boldsymbol{X}}_{\mathbf{3}}$ & $-\tilde{\boldsymbol{X}}_{\mathbf{4}}$ & $\tilde{\boldsymbol{R}}_{\mathbf{1}}$ & $\tilde{\boldsymbol{R}}_{\mathbf{2}}$ & $\tilde{\boldsymbol{R}}_{\mathbf{3}}$ & $\tilde{\boldsymbol{R}}_{\mathbf{4}}$ \\
\hline$\tilde{X}_{1}$ & 1 & 0.2 & -0.2 & 0.2 & -0.2 & -0.2 & -0.2 & -0.2 \\
$-\tilde{\boldsymbol{X}}_{2}$ & 0.2 & 1 & 0 & 0 & 0 & 0 & 0 & 0 \\
$-\tilde{\boldsymbol{X}}_{3}$ & -0.2 & 0 & 1 & 0 & 0 & 0 & 0.2 & 0.2 \\
$-\tilde{\boldsymbol{X}}_{4}$ & 0.2 & 0 & 0 & 1 & -0.2 & -0.2 & 0 & 0
\end{tabular}

Thus

$$
\begin{gathered}
\mu^{\prime}=(0.5,0.8,1,1.5,0.01,0.02,0.10,0.08) \\
\Sigma=\left[\begin{array}{cccccclll}
6.25 & 1.6 & -2 & 2 & -0.02 & -0.03 & -0.1 & -0.1 \\
1.6 & 10.24 & 0 & 0 & 0 & 0 & 0 & 0 \\
-2 & 0 & 16 & 0 & 0 & 0 & 0.16 & 0.16 \\
2 & 0 & 0 & 16 & -0.032 & -0.048 & 0 & 0 \\
-0.02 & 0 & 0 & -0.032 & 0.0016 & 0.00216 & 0.0032 & 0.0032 \\
-0.03 & 0 & 0 & -0.048 & 0.00216 & 0.0036 & 0.0048 & 0.0048 \\
-0.1 & 0 & 0.16 & 0 & 0.0032 & 0.0048 & 0.04 & 0.016 \\
-0.1 & 0 & 0.16 & 0 & 0.0032 & 0.0048 & 0.016 & 0.04
\end{array}\right]
\end{gathered}
$$

and it is easily seen that the unconstrained solution

$$
X=c \Sigma^{-1} \underline{\mu}
$$

is a solution which satisfies the conditions $\alpha_{i} \in[0,1]$ for $i=1,2, \ldots, 4$.

\begin{tabular}{|c|c|c|c|}
\hline Insurance Subportfolio & Retention $\alpha_{i}$ & Expected Profit $\alpha_{i} \ell_{i}$ & $\begin{array}{c}\text { Contribution to overall } \\
\text { Variance } \operatorname{Cov}\left(-\alpha_{i} \tilde{X}_{i}, \tilde{\Delta} u\right)\end{array}$ \\
\hline Motor & 1 & 0.5 & 4.47 \\
\hline Homeowners & 0.54 & 0.43 & 3.87 \\
\hline Industrial Fire & 0.44 & 0.44 & 3.93 \\
\hline GTPL & 0.81 & 1.21 & 10.82 \\
\hline Asset Category & Amount invested $A_{j}$ & Expected Profit $A_{j} R_{j}$ & $\begin{array}{l}\text { Contribution to overall } \\
\text { Variance } \operatorname{Cov}\left(A_{j} \tilde{R}, \tilde{\Delta} u\right)\end{array}$ \\
\hline Medium bond & -69.3 & -0.69 & -6.20 \\
\hline Long bond & 77.9 & 1.56 & 13.92 \\
\hline Equities & 15.9 & 1.59 & 14.21 \\
\hline \multirow[t]{2}{*}{ Real estate } & 8.5 & $\underline{0.68}$ & 6.04 \\
\hline & & $\overline{5.72}$ & 51.07 \\
\hline
\end{tabular}
Choosing $c$ in such a way as to maximize the amount of business retained by the company we obtain the following optimal solution 
The risk return ratio is 0.80 , the amount of net invested assets is 33.0 and the amount of net loss reserves is 98.3 .

By perfect asset liability matching and by investing the equity into the risk free asset one can fully eliminate the asset risk. The vector of expected returns and the covariance matrix of the pure insurance risk are respectively

$$
\underline{\mu_{0}^{\prime}}=(0.5,0.8,1,1.5)
$$

and

$$
\Sigma_{0}=\left[\begin{array}{llrr}
6.25 & 1.6 & -2 & 2 \\
1.6 & 10.24 & 0 & 0 \\
-2 & 0 & 16 & 0 \\
-2 & 0 & 0 & 16
\end{array}\right]
$$

and from the theorem of section 4.2 we know that the maximum risk return ratio which can be achieved in such a situation is

$$
r=\left(\underline{\mu_{0}^{\prime}} \Sigma^{-1} \underline{\mu_{0}}\right)^{1 / 2}=0.53
$$

which is considerably lower than risk return ratio obtained above. Thus, in this example too, it is seen that the assumption of asset risk leads to a considerable improvement of the risk return ratio of the portfolio.

Through quota share cessions the company has reduced the expected profit of its insurance portfolio from 3.8 to 2.58 , i.e. it forgoes a substantial amount of profit in order to maximize its risk return ratio. As a comparison, we now look at the optimal portfolio assuming that the company cedes no quota share. In that case, we have the following vector of expected returns

$$
\mu_{1}^{\prime}=(3.8,0.01,0.02,0.1,0.08)
$$

and covariance matrix

$$
\Sigma_{1}=\left[\begin{array}{cccll}
51.69 & -0.052 & -0.078 & 0.06 & 0.06 \\
-0.052 & 0.0016 & 0.00216 & 0.0032 & 0.0032 \\
-0.078 & 0.00216 & 0.0036 & 0.0048 & 0.0048 \\
0.06 & 0.0032 & 0.0048 & 0.04 & 0.016 \\
0.06 & 0.0032 & 0.0048 & 0.016 & 0.04
\end{array}\right]
$$


And the optimal solution excluding quota share cessions is

\begin{tabular}{|c|c|c|c|}
\hline & $\alpha$ resp. $A$ & Expected Profit & $\begin{array}{c}\text { Contribution } \\
\text { to overall Variance }\end{array}$ \\
\hline Insurance Portfolio & 1 & 3.8 & 50.18 \\
\hline medium bond $\left(A_{1}-L\right)$ & -104.2 & -1.04 & -13.75 \\
\hline long bond & 114.0 & 2.28 & 30.10 \\
\hline equities & 21.8 & 2.18 & 28.83 \\
\hline \multirow[t]{2}{*}{ reai estate } & 10.8 & 0.87 & 11.44 \\
\hline & & $\overline{8.09}$ & $\overline{106.79}$ \\
\hline
\end{tabular}

The risk return ratio is now $r=0.78$ which is only slightly lower than the optimal risk return ratio of 0.80 . In practical circumstances an insurance company may prefer the above solution with the much higher expected profit of 8.09 (vs 5.72) to the optimal solution even if this entails a slight decrease of the risk return ratio.

The optimization method we have derived is nevertheless valuable since it provides us with a benchmark, the optimal portfolio, against which to measure any given portfolio.

\section{COMParison with Other Results in Finance Theory}

\subsection{Markowitz's Portfolio Selection Method}

The portfolio selection method presented here is based on the maximization of the same function as is used in the framework of Markowitz's mean variance method. There are however major differences. In the present model the amount of equity $u$ supporting the business can be chosen by the company. The consequences of the introduction of this additional degree of freedom are discussed in section 2.2. The present model allows a simultanious optimization of a portfolio of risky assets and of insurance risks. The major difference between insurance and financial risks is that the latter are easily traded whereas the former are not. Financial risks are standardized securities for which there exist liquid and transparent secondary markets. The transaction costs are very low, the position of the company can be frequently adjusted at virtually no costs. (Hence the conditions $A_{i} \in(-\infty, \infty)$ or $A_{i} \geq 0$.) Insurance risks once taken on can only be traded on the reinsurance market which is neither liquid nor transparent. It is usually not possible to take a short position in an insurance risk. Increasing one's share of a risk beyond $100 \%$ leads to high transaction costs related to the acquisition of new blocks of business. (Hence the conditions $0 \leq \alpha_{i}, \beta_{i} \leq 1, i=1, \ldots, n$.)

A further difference between insurance and asset risks is the fact that the optimization of insurance risks is a two steps process. Whilst it would 
in principle be possible to determine the optimal retention rates $\alpha_{i}$ and $\beta_{i}$ of each policy, this would hardly be a tractable method in practice, given the fact that even a medium sized company has hundreds of thousands of customers who often buy more than one policy from the company. One has therefore to build insurance subportfolios (e.g. along lines of business and customer segments), to optimize those subportfolios individually (e.g. via surplus, and excess of loss reinsurance as illustrated in section 2) and to build an optimal global portfolio via appropriate quota share cessions. The process is therefore a two steps optimization process and the result depends on the sub portfolio structure which has been chosen.

Finally, the optimal portfolio of assets within the overall portfolio of the insurance company strongly depends on the portfolio of insurance risks. This is especially true since the loss reserve risk entails a short position in a bond portfolio. As a consequence, the portfolio of assets which pertains to the optimal overall portfolio is in general very different from the optimal portfolio of assets on a stand alone basis, as derived from Markowitz's method.

\subsection{CAPM}

\subsection{1.}

Each insurance company optimizes its overall portfolio of insurance and asset risks. The optimal portfolio of the company heavily depends on the gross insurance portfolio which varies considerably from company to company. As a consequence the optimal asset portfolios of different companies are not colinear and are different from the optimal asset portfolio according to the CAPM. Thus the optimal asset portfolio of the company is not a market portfolio, as in the CAPM, but a company specific portfolio. Given the weight of insurance companies and pension funds as institutional investors, the above result may explain why empirical evidence does not confirm the CAPM (see H.S. Houthakker and P.J. Williamson, 1996).

\subsection{2.}

A further difference between the CAPM and our general model is the fact that in our model insurance risks command a loading over and above the expected value of the losses they generate and this in spite of the fact that those risks are not market risks and can be diversified away. The reason why individuals are willing to pay such a loading is because they are risk averse and unable to diversify their risk. Closely held corporations are in a similar position. The case of firms with diffuse ownership is more complex. Stockholders and bondholders of such firms can diversify their claims and do not need to buy insurance. There are however other stakeholders such as employees, clients and suppliers who cannot diversify 
their claims. In the absence of insurance, employees and managers for instance would discount their expected future cash flows at a much higher interest rate to reflect the higher risk. It is therefore worthwhile for the firm to buy insurance even if the price is higher than the actuarially fair premium. Different other reasons such as a lowering of expected bankruptcy costs and a lowering of the company's expected tax liabilities also explain why the 'free lunch' enjoyed by insurance companies is consistent with finance theory. For a more detailed discussion of the topic see Mayers and Smith (1982).

\section{2 .3}

In addition to a free lunch insurance companies also enjoy a free loan. The assumption of the yield curve risk as part of the loss reserve risk is tantamount to issuing a bond without having to pay any spread. This allows the company to achieve a higher risk return ratio than would be possible if it could not issue securities or if had to pay a spread.

\subsection{4.}

Both in the case of the CAPM and of our model the separation theorem holds true. The composition of the optimal portfolio follows from objective factors: the expected returns and the covariance between the returns of individual risks. The decision of how much risk to assume, i.e. the choice of a point on the efficient frontier is a subjective decision, which is separate from the selection of the optimal portfolio.

\section{2 .5}

Within the framework of CAPM, the expected return of asset $i\left(R_{i}\right)$ and the expected return of the market portfolio $\left(R_{M}\right)$ satisfy the following relationship

$$
R_{i}-\rho_{0}=\beta_{i} \cdot\left(R_{M}-\rho_{0}\right) \quad \text { with } \quad \beta_{i}=\frac{\operatorname{Cov}\left(\tilde{R}_{i}, \tilde{R}_{M}\right)}{\operatorname{Var}\left(\tilde{R}_{M}\right)}
$$

Within the framework of our model (see example 3 of section 4.2) the following formulae hold true for the optimal portfolio

$$
\begin{gathered}
\ell_{i}=\frac{\operatorname{Cov}\left(-\tilde{X}_{i}, \tilde{\Delta} u\right)}{\operatorname{Var}(\tilde{\Delta} u)} \cdot\left(E(\tilde{\Delta} u)-\rho_{0} u\right) \\
R_{i}-\rho_{0}=\frac{\operatorname{Cov}\left(\tilde{R}_{i}, \tilde{\Delta} u\right)}{\operatorname{Var}(\tilde{\Delta} u)} \cdot\left(E(\tilde{\Delta} u)-\rho_{0} u\right)
\end{gathered}
$$


We can rewrite the CAPM formula as

$$
\frac{\left(R_{i}-\rho_{0}\right) \cdot B_{i}}{\operatorname{Cov}\left(\tilde{R}_{i} \cdot B_{i}, \tilde{R}_{M}\right)}=\frac{R_{M}-\rho_{0}}{\operatorname{Var}\left(\tilde{R}_{M}\right)}
$$

where $B_{i} i=1, \ldots, n$ are the coefficients pertaining to the optimal portfolio, hence

$$
\tilde{R}_{M}=\sum_{i=1}^{n} \tilde{R}_{i} \cdot B_{i} \cdot\left(\sum_{i=1}^{n} B_{i}\right)^{-1} .
$$

On the other hand the formulae in our model can be rewritten as

$$
\begin{gathered}
\frac{\alpha_{i} \cdot \ell_{i}}{\operatorname{Cov}\left(-\alpha_{i} \tilde{X}_{i}, \tilde{\Delta} u\right)}=\frac{E(\tilde{\Delta} u)-\rho_{0} u}{\operatorname{Var}(\tilde{\Delta} u)} \\
\frac{\left(R_{i}-\rho_{0}\right) \cdot B_{i}}{\operatorname{Cov}\left(\tilde{R}_{i} B_{i}, \tilde{\Delta} u\right)}=\frac{E(\tilde{\Delta} u)-\rho_{0} u}{\operatorname{Var}(\tilde{\Delta} u)}
\end{gathered}
$$

In the special case where there are only asset risks we have

$$
\tilde{R}_{M}=\tilde{\Delta} u \cdot\left(\sum B_{i}\right)^{-1}
$$

i.e. the optimal company portfolio and the optimal market portfolio are identical.

Setting $u=\sum B_{i}$, the second formula can be rewritten as

$$
\frac{\left(R_{i}-\rho_{0}\right) \cdot B_{i}}{\operatorname{Cov}\left(\tilde{R}_{i} B_{i}, \tilde{R}_{M}\right)}=\frac{R_{M}-\rho_{0}}{\operatorname{Var}\left(\tilde{R}_{M}\right)}
$$

and it is seen that the formulae of our model are a generalisation of the CAPM formula. Both types of formulae state that the ratio of expected profit to contribution to the overall covariance is the same for each risk. In the case of the CAPM the formula applies to asset risks only, in the case of our model it applies to asset and insurance risks. In the first case, the reference portfolio is the market portfolio, in the second case it is the company portfolio.

\subsubsection{Discount Rates}

\section{Definition}

The rate of return of the company associated with a given value $u$ of net asset value is

$$
\tilde{R}_{u}=\frac{\tilde{\Delta} u}{u}
$$


Thereby $u$ must be at least equal to the amount of net invested assets, i.e.

$$
u \geq u_{0}=\sum_{i=1}^{n} A_{i}-\sum_{i=1}^{m} \alpha_{i} L_{i}
$$

\section{Theorem}

Assuming that the company may issue securities (Assumption 8) and that insurance risks and financial risks are uncorrelated $\left(\operatorname{Cov}\left(\tilde{X}_{j}, \tilde{R}_{i}\right)=0\right.$ all $\left.i, j\right)$ we have

$$
\tilde{R}_{u_{0}}=\tilde{R}_{M}+\frac{\sum_{i=1}^{n} \alpha_{i}\left(E\left(\tilde{X}_{i}\right)+\ell_{i}-\tilde{X}_{i}\right)}{u_{0}}
$$

where $\tilde{R}_{M}$ is the market rate of return for financial risks according to the CAPM and $u_{0}$ is the amount of net invested assets.

\section{Proof}

Under the assumptions of the theorem we have

$$
\tilde{\Delta} u-\rho_{0} u=\sum_{i=1}^{m} \alpha_{i}\left(E\left(\tilde{X}_{i}\right)+\ell_{i}-\tilde{X}_{i}\right)+\sum_{j=1}^{n}\left(\tilde{R}_{j}-\rho_{0}\right) B_{j}
$$

For any $u \geq u_{0}$ (see section 4.2). Hence

$$
R_{u_{0}}=\frac{\tilde{\Delta} u}{u_{0}}=\frac{\sum \tilde{R}_{j} \cdot B_{j}}{\sum B_{j}}+\frac{\sum \alpha_{i}\left(E\left(\tilde{X}_{i}\right)+\ell_{i}-\tilde{X}_{i}\right)}{u_{0}}
$$

since $u_{0}=\sum A_{i}-\sum \alpha_{j} L_{j}=\sum B_{j}$. And since investment risks and insurance risks are uncorrelated

$$
\left(\sum_{j} \tilde{R}_{j} \cdot B_{j}\right) \cdot\left(\sum_{j} B_{j}\right)^{-1}=\tilde{R}_{M}
$$

q.e.d.

\section{Remark}

Under the assumptions of the theorem we have

$$
\tilde{R}_{u}=\frac{u_{0}}{u} \cdot \tilde{R}_{M}+\frac{\sum \alpha_{i}\left(E\left(\tilde{X}_{i}\right)+\ell_{i}-\tilde{X}_{i}\right)}{u}
$$

According to the CAPM, the discount rate associated with $\tilde{R}_{u}=\frac{\tilde{\Delta} u}{u}$ is

$$
\tilde{R}_{d}(u)=\rho_{0}+\frac{\operatorname{Cov}\left(\tilde{R}_{u}, \tilde{R}_{M}\right)}{\operatorname{Var}\left(\tilde{R}_{M}\right)}\left(\tilde{R}_{M}-\rho_{0}\right)
$$


Assuming that insurance risks and investment risks are uncorrelated, we obtain from the above representation of $\tilde{R}_{u}$

$$
\operatorname{Cov}\left(\tilde{R}_{u}, \tilde{R}_{M}\right)=\frac{u_{0}}{u} \cdot \operatorname{Var}\left(\tilde{R}_{M}\right)
$$

We have thus derived the following

\section{Corollary 1}

Under the assumptions of the preceding theorem, the discount rate of the company is

$$
R_{d}(u)=\frac{u_{0}}{u} R_{M}+\left(1-\frac{u_{0}}{u}\right) \rho_{0}
$$

\section{Corollary 2}

The value of the company is

$$
\frac{E(\tilde{\Delta} u)}{R_{d}(u)}=u+\frac{\sum \alpha_{i} \ell_{i}}{R_{d}(u)}
$$

Proof

$$
E(\tilde{\Delta} u)=\rho_{0} u+\sum_{i} \alpha_{i} \ell_{i}+\sum_{j} B_{j}\left(R_{j}-\rho_{0}\right)
$$

and since insurance and investment risks are uncorrelated, we have

$$
\sum_{j} B_{j}\left(R_{j}-\rho_{0}\right)=\left(\sum_{j} B_{j}\right)\left(R_{M}-\rho_{0}\right)=u_{0}\left(R_{M}-\rho_{0}\right)
$$

hence

$$
\begin{aligned}
& E(\tilde{\Delta} u)=u_{0} \cdot R_{M}+\left(u-u_{0}\right) \rho_{0}+\sum \alpha_{i} \ell_{i} \\
& E(\tilde{\Delta} u)=R_{d}(u) \cdot u+\sum \alpha_{i} \ell_{i}
\end{aligned}
$$

which proves the corollary.

q.e.d.

The value of the company is thus the sum of its net asset value (at market prices) and of the goodwill of the company

$$
G=\frac{\sum \alpha_{i} \ell_{i}}{R_{d}(u)}=\frac{\sum \alpha_{i} \ell_{i}}{\frac{u_{0}}{u} R_{M}+\left(1-\frac{u_{0}}{u}\right) \rho_{0}}
$$

The goodwill depends on $u$ and it is easily seen that $G^{\prime}(u)>0$ and $G^{\prime \prime}(u)<0$. 


\section{Remark}

The goodwill

$$
G=\frac{\sum \alpha_{i} \ell_{i}}{R_{d}(u)}
$$

is the maximum value one should be willing to pay for the access to the business, i.e. for the distribution network. It depends on the amount of net asset value which supports the business, since the higher the equity $u$, the more valuable the excess return $\tilde{\Delta} u-\rho_{0} u$.

Assuming that the amount of equity is determined based on the risk tolerance $\tau$ of the owners of the company

$$
u=\frac{1}{\tau} \frac{V}{R}
$$

(where $V=\operatorname{Var}(\tilde{\Delta} u)$ and $R=E(\tilde{\Delta} u)-\rho_{0} u$ for the optimal portfolio), we obtain the following discount rate

$$
R_{d}=\frac{\tau R}{V} \cdot u_{0} R_{M}+\left(1-\frac{\tau R}{V} u_{0}\right) \rho_{0}
$$

and the goodwill of the company is arrived at by plugging this expression into the above formula. And it is seen that the discount factor is an increasing function of the risk tolerance. Hence the goodwill is a decreasing function of the risk tolerance.

\section{Example}

Example 3 of section 4.2 satisfies the conditions of the above theorem. We have

$$
\begin{gathered}
u_{0}=37.4 \\
u=\tau^{-1} \frac{V}{R}=\tau^{-1} \cdot 62.00=248.0, \quad \text { for } \tau=0.25
\end{gathered}
$$

let $\rho_{0}=5 \%$, we have

$$
R_{M}=\frac{20.71}{37.4}+5 \%=60.4 \%
$$

and we obtain

$$
R_{d}=\frac{u_{0}}{u} R_{M}+\left(1-\frac{u_{0}}{u}\right) \rho_{0}=0.151 \cdot 60.4 \%+0.849 \cdot 5 \%=13.37
$$

Hence

$$
G=\frac{\sum \alpha_{i} \ell_{i}}{R_{d}(u)}=\frac{17.8}{0.1337}=133.2
$$




\section{ACKNOWLEDGEMENT}

I am greatly indebted to one of the referees for drawing my attention to a major inconsistency and allowing me to substantially improve the presentation of this article.

\section{REFERENCES}

Houthakker, H.S. and Williamson, P.J. (1996) The Economics of Financial Markets. Oxford University Press.

MAYERS, D. and Smith, C.W., Jr. (1982) On the Corporate Demand for Insurance. Journal of Business, Vol. 55 , no. 2.

PANJer, H.H. et al. (1998) Financial Economics. The Actuarial Foundation.

SCHNIEPER, R. (1997) Capital Allocation and Solvency Testing. SCOR Notes.

Sharpe, W.F. (1970) Portfolio Theory and Capital Markets. McGraw-Hill.

RENÉ SCHNIEPER

Zurich Insurance Company, Reinsurance

P.O. Box, $\mathrm{CH}-8022$ Zurich

Switzerland 